\title{
Effects of external radiation on biased Aharonov-Bohm rings
}

\author{
O. Entin-Wohlman ${ }^{a, b, c, *}$, Y. $\operatorname{Imry}^{d}$, and A. Aharony ${ }^{a, b, *}$ \\ ${ }^{a}$ Materials Science Division, Argonne National Laboratory, Argonne, Illinois 60439, USA \\ ${ }^{b}$ Department of Physics, Ben Gurion University, Beer Sheva 84105, Israel \\ ${ }^{c}$ Albert Einstein Minerva Center for Theoretical Physics \\ at the Weizmann Institute of Science, Rehovot 76100, Israel \\ ${ }^{d}$ Department of Condensed Matter Physics, The Weizmann Institute of Science, Rehovot 76100, Israel
}

(October 30, 2018)

\begin{abstract}
We consider the currents flowing in a solid-state interferometer under the effect of both an Aharonov-Bohm phase and a bias potential. Expressions are obtained for these currents, allowing for electronic or electron-boson interactions, which may take place solely on a quantum dot placed on one of the interferometer arms. The boson system can be out of equilibrium. The results are used to obtain the transport current through the interferometer, and the current circulating around it under the effect of the Aharonov-Bohm flux. The modifications of both currents, brought about by coupling the quantum dot to an incoherent sonic or electromagnetic source, are then analyzed. By choosing the appropriate range of the boson source intensity and its frequency, the magnitude of the interference-related terms of both currents can be controlled.

PACS numbers: 73.23.-b, 72.15.Gd, 71.38.-k, 73.21.La,72.50.+b
\end{abstract}

\section{INTRODUCTION}

Solid-state interferometers, restricted to the mesoscopic scale in order to retain the coherence of conduction electrons,${ }^{1}$ are constructed from narrow waveguides, possibly containing scatterers, for the electronic paths. An Aharonov-Bohm magnetic flux ${ }^{2}$ between the two paths in such interferometers results in a periodic flux-dependence behavior, which stems from interference of the electronic wave-functions. In recent experiments, ${ }^{3-10}$ carried on interferometers connected to several electronic reservoirs, the current passing through the system in response to a voltage difference has been used to investigate coherent transport. These experiments have revived interest in such systems, whose theoretical ${ }^{11-13}$ and experimental ${ }^{14}$ study has begun much earlier. The current experimental set-ups involve a quantum dot (or two ${ }^{8,9}$ ) embedded in the interferometer, aiming at the study of the transmission properties of the former. These experiments have been followed by many theoretical works, exploring the possibility of deducing the transmission phase of a quantum dot from the measured conductance of the interferometer, ${ }^{15-25}$ and investigating its dependence on various interactions.

The interference of the electronic wave functions in an Aharonov-Bohm interferometer also creates a circulating current, which flows even at thermal equilibrium, and even when the ring is isolated (under these conditions it is usually called 'persistent current'). This current has been invoked as early as 1936 by Pauling, ${ }^{26}$ to explain the large orbital magnetic response of $\pi$ electrons moving on a ring in benzene-type molecules, and soon after has been calculated $^{27}$ in terms of the tight-binding model. The analogy between persistent currents and the Josephson effect has been expounded upon in Refs. 28 and 29. Their discussion of the possible realization of a 'normal Josephson current' in small metallic (or semiconductor) rings, in the presence of some disorder, has sparked much interest in this phenomenon and led to a considerable experimental effort to detect it, either by various magnetic response measurements ${ }^{30-35}$ or by optical spectroscopy. ${ }^{36-38}$ At thermal equilibrium, the persistent current is equivalent to the thermodynamic orbital magnetic moment of the electrons. Since it arises from the interference of the electronic wave functions, then, as long as the electrons are phase-coherent, it will survive the presence of moderate static disorder. ${ }^{1,29}$ Recently, most of the theoretical interest in this phenomenon has shifted to studying charge(or spin) fluctuation effects, ${ }^{39-41}$ time-dependent properties and non-equilibrium situations, ${ }^{42-45}$ or electronic interactions. ${ }^{46-50}$ In addition, there have been recently several attempts ${ }^{51-54}$ to relate the phenomenon of persistent current, which is intimately connected to electronic coherence, to the dephasing of electrons at equilibrium due to the coupling with a boson bath.

Here we study the currents flowing around and through an 'open' interferometer, connected to electronic reservoirs, with a quantum dot placed on one of its arms, when the latter is coupled to an external incoherent radiation source. The electronic reservoirs are held at slightly different chemical potentials, such that the voltages are small enough for the system to be in the linear transport

\footnotetext{
*On leave from the School of Physics and Astronomy, Raymond and Beverly Sackler Faculty of Exact Sciences, Tel Aviv University, Tel Aviv 69978, Israel.
} 
regime. The external radiation source, on the other hand, will be taken as being, and possibly driving the system, out of equilibrium, so that its intensity can serve as a 'control parameter' of the currents. In other words, we study the currents when the electrons are also coupled to an incoherent out-of-equilibrium boson source. We take the electronic system to be free of any interactions, except on the quantum dot, where the electrons are coupled to an external source of sonic (or electromagnetic) waves. The system is depicted in Fig. 1.

Although we use the term 'electron-phonon interaction' throughout this paper, our results apply equally, with minor modifications, to the case where the electrons are coupled to an electro-magnetic source, that is, for the electron-photon interaction. In any event, in order to retain the coherence of the electrons, the systems we consider are necessarily confined to size scales small enough so that the electrons stay coherent at the given temperature. At the same time, the strength of the acoustic source is assumed to be such that the additional decoherence due to it is not detrimental. The precise parameter windows in which this can be achieved is sensitive to acoustic (or electromagnetic) mismatch, details of the sample geometries, etc, and hence their calculations are not carried out here. Also, we do not discuss dephasing, but rather, like in Refs. 42 and 45, we concentrate on a non-equilibrium source of bosons.

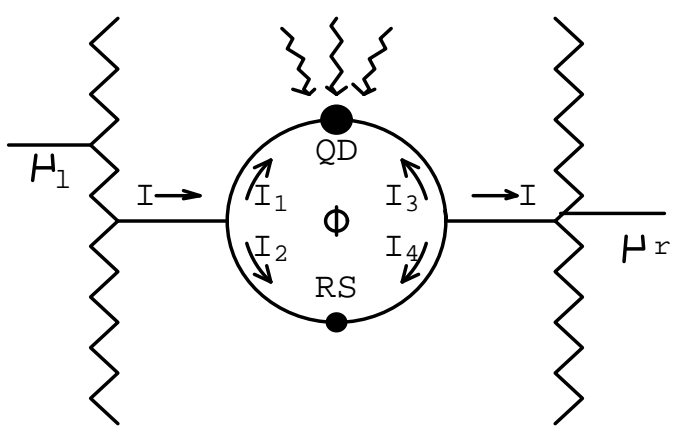

FIG. 1. An Aharonov-Bohm interferometer, containing a quantum dot (QD) on its upper arm and threaded by a magnetic flux $\Phi$. The lower arm of the interferometer contains a 'reference' site (RS). The ring is connected to two electronic reservoirs whose chemical potentials are either equal or have a small difference, allowing a current $I$ to flow from the left to the right. The wavy vectors denote the external beam radiated on the dot.

When the electrons are coupled to a boson source, the naïve expectation is that the coherent current decreases due to loss of coherence, caused by inelastic processes as well as by renormalization effects due to the 'dressing' of the electrons by the bosons (the polaron effect ${ }^{55}$ ). The latter is manifested by an overall Debye-Waller ex- ponent. However, it turns out that this is not the whole effect brought about by the radiation. In the case of isolated rings, it has been found ${ }^{56}$ that when the electrons are coupled to phonons, the persistent current is not only diminished; Rather, there appears an additional term, which originates from delicate resonance processes in which at least two phonons are involved (those were termed 'doubly-resonant processes'). The additional orbital magnetic moment appears at non-zero temperatures, and has a non-monotonic temperature dependence at sufficiently low temperatures. ${ }^{56}$ At thermal equilibrium, this new term has been found to further reduce the persistent current (beyond its value in the absence of the coupling to the bosons). However, at non-equilibrium situations, the magnitude of that 'extra' contribution may be tuned by controlling the intensity of the radiation in a certain frequency range, which is experimentally feasible. Possibly related experiments with extremely interesting results have recently been reported in, e.g., Refs. 57 and 58. Here we examine the effect of the electronphonon coupling on a biased Aharonov-Bohm interferometer, which consists of an 'open' ring, connected to two reservoirs. Then, in addition to the circulating current induced by the magnetic phase, there appears also a transport current. We find that in a certain sense, the open ring is more amenable to manipulations by an external radiation source. We show that both the circulating and the transport currents are affected by a radiation source in a similar manner: Beside the overall Debye-Waller factor, they each acquire an additional contribution. In the case of an open ring, that additional term does not necessitate the existence of real resonant transitions between the initial and the final state, it appears at a lower order in the electron-phonon interaction, (as compared to the situation in isolated rings) and it exists even at zero temperature. The magnitude of that contribution can again be tuned by controlling the intensity of the radiation in a certain frequency range. In other words, by coupling the electrons to an out-of-equilibrium radiation source, one may control both the circulating and the transport current. Such a relation between the radiation intensity and the orbital magnetic moment may open interesting possibilities for future nano-devices.

Our method of calculation is to express all partial currents flowing in the system (i.e., $I_{1}, I_{2}, I_{3}$, and $I_{4}$, see Fig. 1 ) in terms of the exact (and generally, unknown) Green function on the dot, which includes all the effects of the coupling to the interferometer, the external reservoirs, and the interactions taking place on the dot. These expressions do not necessitate a near-equilibrium situation. In so doing, we derive general expressions for the current passing through the interferometer, $I$, and the current circulating around it which is induced by the AharonovBohm flux, in terms of the exact Green function on the dot. We then use these results to investigate the effect of coupling to a boson source on both currents.

We begin in Sec. II by the derivation of the partial currents, the transport current, and the circulating current. 
The expressions we obtain are valid also for the case in which the electrons experience electronic interactions on the dot. In particular, our result for the transport current generalizes the ones reported previously, ${ }^{21,22}$ which were derived under the assumption that there is no scattering on the reference arm. Within that approximation, the flux-dependence of the linewidth on the quantum dot level is lost. This flux dependence, as we show, turns out to be crucial in determining the circulating current. Section II is supplemented by an Appendix, detailing the computation of the partial currents (Appendix A). In sec. III we employ the general result for the transport current to study the effect of the coupling to a boson source. To this end, we use an approximate expression for that dot Green function, ${ }^{59-61}$ for the case in which the electrons on the dot are coupled linearly to a sonic source. Section IV is devoted to the analysis of the circulating current under irradiation. For the sake of completeness, we include in that section a discussion of the effect of electron-phonon coupling on electrons moving on electronically-isolated rings (which are decoupled from the leads). Finally, we summarize our findings in Sec. V.

\section{THE CURRENTS IN A BIASED INTERFEROMETER}

Figure 1 portrays an Aharonov-Bohm interferometer, with a quantum dot placed on its upper arm, and a second electronic site placed on the other arm, serving as a 'reference' site. All interactions (among the electrons, or electron-boson interactions) are taking place only on the quantum dot. The interferometer is connected at the left and at the right to electronic reservoirs, kept at slightly different (or equal) chemical potentials. The connection is via single-channel leads. The model Hamiltonian describing this system consists of four terms,

$$
\mathcal{H}=\mathcal{H}_{\text {leads }}+\mathcal{H}_{\text {ref }}+\mathcal{H}_{\text {d }}+\mathcal{H}_{\text {tun }}
$$

in which the first term describes the leads, which are assumed to be two free-electron systems,

$$
\mathcal{H}_{\text {leads }}=\sum_{k} \epsilon_{k} c_{k}^{\dagger} c_{k}+\sum_{p} \epsilon_{p} c_{p}^{\dagger} c_{p} .
$$

(We omit spin indices when they are not necessary.) The left lead states are denoted by $k$, and the right ones by $p$, with $c_{k}\left(c_{p}\right)$ being the destruction operator for states on the left (right) lead. For one-dimensional leads, described by a tight-binding model with a nearest-neighbor hopping matrix element $J$, one has $\epsilon_{k}=-2 J \cos k$, and similarly $\epsilon_{p}=-2 J \cos p$. The chemical potential in the reservoir connected to the left lead, $\mu_{\ell}$, can differ from that on the right reservoir, $\mu_{r}$. Otherwise, the two leads are taken as identical, i.e., they have the same large bandwidth $2 J$. The reference site is taken for simplicity as having a single localized level, of energy $\epsilon_{0}$; Hence

$$
\mathcal{H}_{\text {ref }}=\epsilon_{0} c_{0}^{\dagger} c_{0}
$$

The dot Hamiltonian $\mathcal{H}_{\mathrm{d}}$ is not specified at the moment; It may include electron-electron interactions or electronphonon interactions. For simplicity, we assume that only one of the dot single-energy levels is effectively connected to the leads. It is possible to carry out a more general calculation; However, the algebra then becomes complicated and may obscure the physical effects we wish to explore. Hence we write for the tunneling Hamiltonian

$$
\begin{aligned}
\mathcal{H}_{\text {tun }} & =\sum_{k} V_{k} c_{k}^{\dagger} d+\sum_{p} V_{p} c_{p}^{\dagger} d \\
& +\sum_{k} v_{k} c_{k}^{\dagger} c_{0}+\sum_{p} v_{p} c_{p}^{\dagger} c_{0}+h c
\end{aligned}
$$

where $d$ is the destruction operator for the electron on the dot. The tunneling matrix elements for a onedimensional tight-binding model read

$$
\begin{aligned}
& V_{k}=-\sqrt{\frac{2}{N}} j_{\ell} \sin k, \quad V_{p}=-\sqrt{\frac{2}{N}} j_{r} \sin p \\
& v_{k}=-\sqrt{\frac{2}{N}} i_{\ell} e^{i \phi_{\ell}} \sin k, v_{p}=-\sqrt{\frac{2}{N}} i_{r} e^{-i \phi_{r}} \sin p
\end{aligned}
$$

where $N$ is the number of sites on each of the leads, and gauge invariance allows one to assign the flux dependence to the reference arm, such that the total flux (which is the magnetic flux threading the ring, measured in units of the flux quantum) is

$$
\Phi=\phi_{\ell}+\phi_{r}
$$

In Eq. (5), $j_{\ell}$ and $j_{r}$ are the matrix elements coupling the dot to the left and right point contacts, and $i_{\ell}$ and $i_{r}$ are those connecting the reference site to the same points. We emphasize that the model considered here does not allow $^{24}$ for any electron losses. This is often referred to as a "closed interferometer".

Under the circumstances described above, a transport current $I$ is passed through the ring, say from left to right. This current splits into the currents moving in the upper and lower arms of the ring,

$$
I=I_{1}+I_{2}
$$

When all electrons entering the interferometer from the left reservoir leave it into the right one, and are not lost to the surrounding, (as sometimes happens in the experiments), one has $I_{1}+I_{3}=I_{2}+I_{4}=0$. For reasons related to the detailed calculations below, we keep the four partial currents separately. The current circulating the ring under the effect of the Aharonov-Bohm flux, $I_{c i r}$, is conveniently defined as

$$
2 I_{c i r}=\left.\frac{1}{2}\left(I_{1}-I_{2}\right)\right|_{\Phi}-\left.\frac{1}{2}\left(I_{1}-I_{2}\right)\right|_{-\Phi},
$$


in order to avoid spurious currents caused by geometrical asymmetries. It is therefore seen that the calculation of both the transport current and the circulating one requires the knowledge of the partial currents in the interferometer.

An efficient way to find those currents is to employ the Keldysh technique, which is particularly suitable to handle non-equilibrium situations. ${ }^{62}$ Using the Keldysh notations, the partial currents $I_{1}$ and $I_{2}$ are given by ${ }^{61}$ (in units in which $\hbar=1$ )

$$
\begin{aligned}
& I_{1}=e \int \frac{d \omega}{2 \pi} \sum_{k} V_{k}\left(G_{k d}^{<}(\omega)-G_{d k}^{<}(\omega)\right), \\
& I_{2}=e \int \frac{d \omega}{2 \pi} \sum_{k}\left(v_{k}^{*} G_{k 0}^{<}(\omega)-v_{k} G_{0 k}^{<}(\omega)\right),
\end{aligned}
$$

where

$$
G_{a b}^{<}(\omega)=\int d t e^{i \omega t} i\left\langle b^{\dagger} a(t)\right\rangle
$$

and the operators $a$ and $b$ stand for $c_{k}, c_{p}, c_{0}$, or $d$. The other two partial currents, $I_{3}$ and $I_{4}$, are derived from Eqs. (9) by changing the lead index $k$ into the second lead index, $p$.

The computation of all four partial currents is detailed in Appendix A. Here we summarize the results. The first step taken there is to obtain explicit expressions [see Eqs. (A27) and (A34)] for the partial currents in terms of the various parameters, and the exact Green function on the dot, which includes all effects of interactions, as well as the couplings to the interferometer, to the electronic reservoirs and to the phonon source. In the Keldysh technique this means that the above-mentioned expressions include the Keldysh function $G_{d d}^{<}$, [see Eq. (10)], and the usual retarded $\left(G_{d d}^{R}\right)$ and the advanced $\left[G_{d d}^{A}=\left(G_{d d}^{R}\right)^{*}\right]$ dot Green functions. The frequency $(\omega)$ - integration of the former, $\int d \omega G_{d d}^{<}$, has a very clear physical meaning: It gives the occupation number of the electrons on the dot, $n_{d}$.

When the interferometer is biased, the Keldysh Green function $G_{d d}^{<}$and the occupation $n_{d}$ are affected by the voltage difference, such that current conservation, $I_{1}+I_{3}=0$, is ensured (see Fig. 1). In practice, however, the calculation of the Keldysh function is not simple (except for the interaction-free system). We therefore resort to an approximation, which gives it in terms of $G_{d d}^{R}$ and $G_{d d}^{A}$. Explicitly, one finds (see Appendix A2 for details)

$$
\begin{aligned}
I_{1}+I_{3} & =e \int \frac{d \omega}{2 \pi}\left[\left(\Sigma_{\mathrm{ext}}^{R}-\Sigma_{\mathrm{ext}}^{A}\right) G_{d d}^{<}\right. \\
& \left.+\Sigma_{\mathrm{ext}}^{<}\left(G_{d d}^{A}-G_{d d}^{R}\right)\right],
\end{aligned}
$$

where the frequency dependence of the various functions is suppressed for brevity. Here, $\Sigma_{\text {ext }}^{R}$ is that part of the retarded self-energy on the dot, which comes solely from the couplings to the interferometer and to the leads.
Namely, it is the self-energy part for the interaction-free system. Similarly, $\Sigma_{\text {ext }}^{A}=\left(\Sigma_{\text {ext }}^{R}\right)^{*}$ is the advanced selfenergy coming from those couplings, and $\Sigma_{\text {ext }}^{<}$is the corresponding Keldysh function. All the above three selfenergies can be found quite straightforwardly, as they pertain to the non-interacting parts of the Hamiltonian [see Eqs. (A37) and (A38)] . When the system is free of interactions, or when it is un-biased, namely, $\mu_{\ell}=\mu_{r}$ (see Appendix A2), the integrand in Eq. (11) vanishes. When the (interacting) system is slightly biased, the dot Green functions are not known exactly. However, the finite bias causes only very small changes in the Fermi functions $f_{\ell}$ and $f_{r}$, of the left and of the right reservoirs, except in the range $\mu_{\ell}-\mu_{r}$ around the Fermi energy. Here,

$$
f_{\ell}(\omega)=\frac{1}{e^{\beta\left(\omega-\mu_{\ell}\right)}+1}, \quad f_{r}(\omega)=\frac{1}{e^{\beta\left(\omega-\mu_{r}\right)}+1} .
$$

Hence, we expect the integrand in Eq. (11) to be dominated by contributions from that vicinity of the Fermi energy. If the integrand in Eq. (11) varies slowly with the frequency there, then the vanishing of the integral would also imply the vanishing of the integrand, namely,

$$
G_{d d}^{<}=\Sigma_{\text {ext }}^{<} \frac{G_{d d}^{R}-G_{d d}^{A}}{\Sigma_{\text {ext }}^{R}-\Sigma_{\text {ext }}^{A}} .
$$

In some cases, 61 this equation follows from the "wideband-approximation' which neglects the $\omega$-dependence of the resonance width $\Im \Sigma_{\text {ext }}^{A}$. Equation (13) is used to eliminate the dot Keldysh Green function from the expressions for the currents. It should be emphasized (see Appendix A4) that the relationship Eq. (13) is exact for the un-biased system. This point is important for the calculation of the persistent current, for which one has to keep the contributions of all frequencies. We note in passing that the sum $I_{2}+I_{4}$ vanishes identically as checked by an explicit calculation.

The next step taken in Appendix A is to employ the partial currents in order to obtain the transport current [Eq. (7)] and the persistent current [Eq. (8)]. The former is obtained using the wide-band approximation, in which the frequency-dependence of the self-energies is suppressed (see Appendix A3 for details),

$$
\begin{aligned}
I= & e \int \frac{d \omega}{2 \pi}\left(f_{r}-f_{\ell}\right)\left\{T _ { B } \left(1+G_{d d}^{R} \Sigma_{\mathrm{ext}}^{R}\right.\right. \\
+ & \left.G_{d d}^{A} \Sigma_{\mathrm{ext}}^{A}+\Sigma_{\mathrm{ext}}^{R} \Sigma_{\mathrm{ext}}^{A} \frac{G_{d d}^{R}-G_{d d}^{A}}{\Sigma_{\mathrm{ext}}^{R}-\Sigma_{\mathrm{ext}}^{A}}\right) \\
+ & 4 \Gamma_{\ell} \Gamma_{r} X_{B} \frac{G_{d d}^{R}-G_{d d}^{A}}{\Sigma_{\mathrm{ext}}^{R}-\Sigma_{\mathrm{ext}}^{A}}+\sqrt{T_{B} \Gamma_{\ell} \Gamma_{r} X_{B}} 2 \cos \Phi \\
& \left.\times\left(G_{d d}^{R}+G_{d d}^{A}+\left(\Sigma_{\mathrm{ext}}^{R}+\Sigma_{\mathrm{ext}}^{A}\right) \frac{G_{d d}^{R}-G_{d d}^{A}}{\Sigma_{\mathrm{ext}}^{R}-\Sigma_{\mathrm{ext}}^{A}}\right)\right\} .
\end{aligned}
$$

The transport current consists of three parts: The first term in the curly brackets of Eq. (14) is the current flowing through the reference arm (the lower arm of the interferometer in Fig. 1), 'dressed' by the processes in 
which the electrons travel around the ring, as is manifested by the appearance of the dot Green functions. Here, $T_{B}$ [see Eq. (A56)] is the transmission coefficient of the reference branch, when the upper arm of the interferometer is cut off. The second term in the curly brackets of Eq. (14) is the current flowing through the interferometer arm containing the quantum dot; Here $\Gamma_{\ell}$ and $\Gamma_{r}$ [see Eq. (A54)] are the partial linewidths on the dot, caused by the couplings to the leads, and

$$
X_{B}=1-T_{B} \frac{\left(\gamma_{\ell}+\gamma_{r}\right)^{2}}{4 \gamma_{\ell} \gamma_{r}}
$$

where $\gamma_{\ell}$ and $\gamma_{r}$ [see Eq. (A53)] are the partial linewidths on the reference site, caused by the couplings to the leads. (Note that when these are symmetric, $\gamma_{\ell}=\gamma_{r}, X_{B}$ becomes equal to the reflection coefficient of the reference branch, $R_{B}=1-T_{B}$.) The last term in Eq. (14) results directly from interference, since it necessitates transmission through both arms of the interferometer, as is manifested by the product $\sqrt{T_{B} \Gamma_{\ell} \Gamma_{r}}$ there.

Several comments on the result (14) are called for: 1 . The transport current $I$ is even in the Aharonov-Bohm flux as it should be, obeying the Onsager symmetry. ${ }^{63}$ This happens because ${ }^{24}$ the dot Green function $G_{d d}^{R}$ and $\Sigma_{\text {ext }}^{R}$ are even functions of $\Phi$, due to additive contributions (with equal amplitudes) from clockwise and counterclockwise motions of the electron around the ring. 2 . When the electronic system is free of interactions, the 'external' self-energy part $\Sigma_{\text {ext }}$ constitutes the entire selfenergy of the dot Green function, namely,

$$
G_{d d}^{R 0}=\frac{1}{\omega-\epsilon_{d}-\Sigma_{\mathrm{ext}}^{R}},
$$

where $\epsilon_{d}$ is the energy of the localized level on the dot, and the superscript ' 0 ' denotes the absence of interactions. In that case $\left(G_{d d}^{R 0}-G_{d d}^{A 0}\right) /\left(\Sigma_{\text {ext }}^{R}-\Sigma_{\text {ext }}^{A}\right)=G_{d d}^{R 0} G_{d d}^{A 0}$, and the transport current becomes

$$
I^{0}=e \int \frac{d \omega}{2 \pi}\left(f_{r}(\omega)-f_{\ell}(\omega)\right) T^{0}(\omega) \simeq \frac{e^{2}}{2 \pi} T^{0}(0) V,
$$

where $V$ is the potential difference on the interferometer, and $T^{0}$ is the transmission coefficient of the noninteracting ring,

$$
\begin{aligned}
T^{0}(\omega)= & \left|G_{d d}^{R 0}(\omega)\right|^{2} \\
& \times\left|\sqrt{T_{B}}\left(\omega-\epsilon_{d}\right) e^{i \Phi}+2 \sqrt{\Gamma_{\ell} \Gamma_{r} X_{B}}\right|^{2} .
\end{aligned}
$$

Equation (18) resembles the two-slit formula, as it consists of the absolute value squared of the sum of two terms: The one related to the transmission amplitude of the reference arm (having the factor $\sqrt{T_{B}}$ ) and the other which is related to the transmission amplitude through the dot (as expressed by $\sqrt{\Gamma_{\ell} \Gamma_{r}}$ ), with the AharonovBohm phase factor multiplying one of those. However, in contrast to the two-slit formula, here both terms are real, resulting in an expression which is even in the flux. This aspect of the transmission has been discussed in great detail in Refs. 24 and 25. In the next section, we find that it persists also when the electrons on the dot are exposed to external radiation. 3. For general values of the flux $\Phi$, the interaction-free transmission (18) does not show the Fano anti-resonances, at which the transmission vanishes (although the line-shape will be asymmetric). The reason is that when $\Phi \neq 0$ or $\Phi \neq \pi$, the interference between the two arms of the interferometer can never be made completely destructive, as was noted in Ref. 64 . On the other hand, finite values of the flux do not prevent the transmission from achieving the unitary limit. Inspection of Eq. (18) in conjunction with the explicit expressions for the external self-energy, Eqs. (A59), shows that the maximal value for the transmission, $T^{0}=1$, is reached when the interferometer is symmetric, i.e., when $\Gamma_{\ell}=\Gamma_{r}$ and $\gamma_{\ell}=\gamma_{r}$, the local level on the dot becomes a resonance, i.e., $\omega-\epsilon_{d}-\Re \Sigma_{\text {ext }}^{R}=0$, and the Aharonov-Bohm flux takes the particular value $\cos \Phi=-T_{B} /\left(1+R_{B}\right)$.

We next turn to the computation of the circulating current in an open ring, Eq. (8). In the case of noninteracting electrons, that current has been the subject of several studies. ${ }^{13,65-67}$ Here we generalizes those calculations to the case where the electrons experience interactions on the quantum dot.

Inserting the expressions for the partial currents into Eq. (8) (see Appendix A4 for details) we find that the circulating current consists of two contributions. The first one, [see Eq. (A69)], is related to the sum of the two electronic distributions, $f_{\ell}+f_{r}$. It therefore flows even when the interferometer is un-biased, and $f_{\ell}=f_{r}$. The second contribution, [see Eq. (A70)], arises only when the system is biased, being related to the difference $f_{\ell}-f_{r}$, and only when, in addition, the couplings of the dot and/or the couplings of the reference site to the interferometer are not equal, namely, when $i_{\ell} \neq i_{r}$ and/or $j_{\ell} \neq j_{r}$ [see Eqs. (5)]. Both contributions are induced by the Aharonov-Bohm flux and hence are proportional to $\sin \Phi$. However, the second term seems to be not as interesting as the first. We therefore omit any further consideration of that part of the circulating current, and focus only the first contribution, which reads ${ }^{68}$

$$
I_{p c}=e \int \frac{d \omega}{i \pi} \frac{f_{\ell}+f_{r}}{4}\left[\frac{\partial \Sigma_{\mathrm{ext}}^{R}}{\partial \Phi} G_{d d}^{R}-c c\right] .
$$

It is interesting to note that Eq. (19) averages the fluxderivative of the external self-energy over energy, with weights containing the densities of electrons and singleparticle states with that energy (which are contained in $\left.G_{d d}\right)$. The flux-derivative of $G_{d d}$ does not appear. This is reminiscent of the equilibrium case, where the persistent current is given by the flux-derivative of the energies, weighed by the electronic populations, without the appearance of the flux-derivative of those, (see, for example, Ref. 56). Since both $\Sigma_{\text {ext }}^{R}$ and $G_{d d}^{R}$ are even in $\Phi$, $I_{p c}$ is odd in $\Phi$, as it should. 
It is sometimes useful to discuss the properties of an open electronic system in the language of scattering theory, ${ }^{65,67}$ employing the concept of 'transmission phases', or the Friedel phase. Such a description is particularly useful in the case of interaction-free electrons. Indeed, by manipulating Eqs. (16) and (19), one obtains that the persistent current of such a system, $I_{p c}^{0}$, is given by

$$
I_{p c}^{0}=e \int \frac{d \omega}{\pi} \frac{f_{\ell}(\omega)+f_{r}(\omega)}{2} \frac{\partial \delta^{0}(\omega)}{\partial \Phi}
$$

where $\delta^{0}$ is the phase of the retarded Green function $G^{R 0}=\left(G^{A 0}\right)^{*}$,

$$
\tan \delta^{0}(\omega)=-\frac{\Im \Sigma_{\mathrm{ext}}^{R}}{\omega-\epsilon_{d}-\Re \Sigma_{\mathrm{ext}}^{R}} .
$$

Hence, in a steady-state situation, the persistent current of non-interacting electrons is related to the variation of the transmission-phase with the Aharonov-Bohm flux. (See Ref. 66 for a different derivation of this result.) This variation replaces the variation of the eigen-energies with the flux in the equilibrium situation as the origin of the persistent current. ${ }^{56}$

\section{RADIATION EFFECTS ON THE TRANSPORT CURRENT}

The coupling between the electrons residing on the dot and a sonic source may be described by a linear, local, electron-phonon interaction, ${ }^{55}$

$$
\mathcal{H}_{\mathrm{el}-\mathrm{ph}}=\sum_{\mathbf{q}} \alpha_{\mathbf{q}}\left(b_{\mathbf{q}}^{\dagger}-b_{\mathbf{q}}\right) d^{\dagger} d
$$

in which $\alpha_{\mathbf{q}}=-\alpha_{-\mathbf{q}}=-\alpha_{\mathbf{q}}^{*}$ is the electron-phonon coupling and $b_{\mathbf{q}}^{\dagger}$ is the creation operator for the boson of wavevector $\mathbf{q}$. To study the transport current in the presence of such an interaction, one has to add $\mathcal{H}_{\mathrm{el}-\mathrm{ph}}$ to the Hamiltonian Eq. (1), together with the free Hamiltonian of the boson excitations, to compute the dot Green function, and then to use it in Eq. (14). In the case of a linear electron-phonon interaction, one is able to obtain an approximate form for the Green function $G_{d d}$, by assuming that the external self-energy does not depend on the frequency. ${ }^{59,60}$ (For a numerical solution in the presence of an equilibrium phonon source, see Ref. 69.) This is a valid approximation, since the small potential difference, temperature, etc., restrict the frequency-integration in Eq. (14) to a narrow range around the common Fermi energy of the two reservoirs. The explicit expression for $\Sigma_{\text {ext }}^{R}$, valid for the case of an Aharonov-Bohm interferometer, is given in Eq. (A59).

The Green function of the dot, which takes into account the electron-phonon coupling (22), was found in Refs. 59, 60, and 61. Here we extend their result to include the effect of the reference arm and to allow for a finite electronic occupation, $n_{d}$, on the dot. The resulting form is then

$$
\begin{aligned}
G_{d d}^{R}(\omega)= & -i K\left[\left(1-n_{d}\right) \int_{0}^{\infty} d t e^{i\left(\omega-\epsilon_{d}-\Sigma_{\mathrm{ext}}^{R}\right) t} e^{\Psi(t)}\right. \\
& \left.+n_{d} \int_{0}^{\infty} d t e^{i\left(\omega-\epsilon_{d}-\Sigma_{\mathrm{ext}}^{R}\right) t} e^{\Psi(-t)}\right]
\end{aligned}
$$

In the non-equilibrium case, $n_{d}$ is determined by both the acoustic intensity and the relaxation processes. The on-site energy on the dot, $\epsilon_{d}$, is now renormalized by the polaron shift, $\epsilon_{P}=\sum_{\mathbf{q}}\left|\alpha_{\mathbf{q}}\right|^{2} / \omega_{q}$, where $\omega_{q}$ denotes the phonon frequency. Since this renormalization is temperature- and flux-independent, it will be omitted. The other phonon variables are contained in $K$, the Debye-Waller factor, and in $\Psi(t)$. Explicitly,

$$
\begin{aligned}
K & =\exp \left[-\sum_{\mathbf{q}} \frac{\left|\alpha_{\mathbf{q}}\right|^{2}}{\omega_{q}^{2}}\left(1+2 N_{q}\right)\right], \\
\Psi(t) & =\sum_{\mathbf{q}} \frac{\left|\alpha_{\mathbf{q}}\right|^{2}}{\omega_{q}^{2}}\left[N_{q} e^{i \omega_{q} t}+\left(1+N_{q}\right) e^{-i \omega_{q} t}\right],
\end{aligned}
$$

where $N_{q}=\left\langle b_{\mathbf{q}}^{\dagger} b_{\mathbf{q}}\right\rangle$ is the phonon occupation of the $\mathbf{q}$ mode, which is not necessarily the thermal equilibrium one, but may be tuned externally.

Perhaps the simplest way to access the effect of the acoustic coupling is by expanding $G_{d d}$ in the electronphonon coupling $\left|\alpha_{\mathbf{q}}\right|^{2}$,

$$
G_{d d}^{R}(\omega)=K G_{d d}^{R 0}(\omega)+\sum_{s= \pm} \sum_{\mathbf{q}} C_{\mathbf{q}}^{s} G_{d d}^{R 0}\left(\omega+s \omega_{q}\right)
$$

where the interaction-free Green function $G_{d d}^{R 0}$ is given in Eq. (16). Here, $s= \pm$, and

$$
C_{\mathbf{q}}^{+}=\frac{\left|\alpha_{\mathbf{q}}\right|^{2}}{\omega_{q}^{2}}\left(N_{q}+n_{d}\right), C_{\mathbf{q}}^{-}=\frac{\left|\alpha_{\mathbf{q}}\right|^{2}}{\omega_{q}^{2}}\left(1+N_{q}-n_{d}\right) .
$$

For a weak electron-phonon coupling, the Debye-Waller factor is

$$
K \simeq 1-\sum_{s= \pm} \sum_{\mathbf{q}} C_{\mathbf{q}}^{s}
$$

However, it is instructive to keep the Debye-Waller factor $K$, which multiplies the zero-order term in the expansion (25) (and, in principle, all other terms in the expansion) in its implicit form, in order to demonstrate its role in diminishing all contributions to the current, and not only those arising from interference. ${ }^{70}$

It is thus seen that the dot Green function in the presence of the electron-phonon coupling may be written as a series of terms in which there appear the interaction-free Green functions, with their frequency argument shifted by the phonon frequencies, ${ }^{71,72}$ each multiplied by the relevant phonon occupation numbers. Hence, it is quite obvious that the transport current will have a similar 
form. Indeed, upon inserting the result (25) into the expression for the transport current, Eq. (14), one finds

$$
I=e \int \frac{d \omega}{2 \pi}\left(f_{r}(\omega)-f_{\ell}(\omega)\right) T^{\mathrm{rad}}(\omega),
$$

in which the transmission of the irradiated interferometer, $T^{\mathrm{rad}}$, is

$$
T^{\mathrm{rad}}(\omega)=K T^{0}(\omega)+\sum_{s= \pm} \sum_{\mathbf{q}} C_{\mathbf{q}}^{s} T^{0}\left(\omega+s \omega_{q}\right),
$$

and the interaction-free transmission is given in Eq. (18). It is seen that the processes contained in $T^{0}\left(\omega+s \omega_{q}\right)$, compensate partially for the detrimental effect of the Debye-Waller factor, $K$. We will encounter a similar situation in the discussion of the circulating current. Since we are operating in the linear response regime, it suffices to study the result (29) at the Fermi energy, namely, at zero frequency in our notations.

Let us first consider the radiation effect on the transport through the ring in the unitary limit, namely when $T^{0}(0)=1$. This situation, as mentioned above, occurs for a symmetric ring, when $\epsilon_{d}+\Re \sum_{\text {ext }}^{R}=0$ and $\cos \Phi=-T_{B} /\left(1+R_{B}\right)$. Under these conditions

$$
\left.T^{0}\left(s \omega_{q}\right)\right|_{\mathrm{res}}=1-R_{B} \frac{\omega_{q}^{2}}{\left(\Im \Sigma_{\mathrm{ext}}^{R}\right)^{2}+\omega_{q}^{2}} .
$$

Inserting this into Eq. (29), and using Eq. (27), yields

$$
\begin{aligned}
& \left.T^{\mathrm{rad}}(0)\right|_{\mathrm{res}}=1-R_{B} \sum_{s= \pm} \sum_{\mathbf{q}} C_{\mathbf{q}}^{s} \frac{\omega_{q}^{2}}{\left(\Im \sum_{\text {ext }}^{R}\right)^{2}+\omega_{q}^{2}} \\
= & 1-R_{B} \sum_{\mathbf{q}} \frac{\left|\alpha_{\mathbf{q}}\right|^{2}}{\omega_{q}^{2}}\left(1+2 N_{q}\right) \frac{\omega_{q}^{2}}{\left(\Im \Sigma_{\text {ext }}^{R}\right)^{2}+\omega_{q}^{2}} .
\end{aligned}
$$

At resonance, the transmission is independent of the electronic occupation on the dot. The coupling with the bosons reduces the transmission at resonance, the more so as the intensity of the boson source in a certain frequency range increases; It is interesting to note, however, that this effect becomes smaller as the reflection coefficient of the reference arm decreases (and therefore the current tends to go mainly through that arm).

To study the effect of the radiation in the general case, it is convenient to present the transmission $T^{\mathrm{rad}}$ in the form

$$
\begin{aligned}
T^{\mathrm{rad}}(0) & =T^{0}(0)+\frac{1}{2} \sum_{\mathbf{q}} A_{\mathbf{q}}^{-}\left[T^{0}\left(\omega_{q}\right)-T^{0}\left(-\omega_{q}\right)\right] \\
& +\frac{1}{2} \sum_{\mathbf{q}} A_{\mathbf{q}}^{+}\left[T^{0}\left(\omega_{q}\right)+T^{0}\left(-\omega_{q}\right)-2 T^{0}(0)\right],
\end{aligned}
$$

where $-2 T^{0}(0)$ comes from the Debye-Waller factor. Here $A_{\mathbf{q}}^{+}$is directly proportional to the radiation intensity, while $A_{\mathbf{q}}^{-}$does not depend on it. Explicitly,

$$
\begin{aligned}
& A_{\mathbf{q}}^{+}=C_{\mathbf{q}}^{+}+C_{\mathbf{q}}^{-}=\frac{\left|\alpha_{\mathbf{q}}\right|^{2}}{\omega_{q}^{2}}\left(1+2 N_{q}\right), \\
& A_{\mathbf{q}}^{-}=C_{\mathbf{q}}^{+}-C_{\mathbf{q}}^{-}=\frac{\left|\alpha_{\mathbf{q}}\right|^{2}}{\omega_{q}^{2}}\left(2 n_{d}-1\right) .
\end{aligned}
$$

Equation (32) shows that by shining a beam of bosons at a certain frequency range, the transport current increases linearly with the intensity of the beam, as long as the latter is not too large. For example, when the interferometer is far from resonance, namely when $\left|\epsilon_{d}\right| \gg \Gamma_{0}$, where $\Gamma_{0}=\Gamma_{\ell}+\Gamma_{r},\left(\Gamma_{0}\right.$ is the width of the resonance level of the quantum dot itself, when it is disconnected from the reference arm), we find that the transmission, to lowest order in $\Gamma_{0} /\left|\epsilon_{d}\right|$ becomes

$$
\begin{aligned}
& \left.T^{\mathrm{rad}}(0)\right|_{\text {off res }}=T_{B}-\sqrt{T_{B} R_{B}}\left(T_{B}+\left(1+R_{B}\right) \cos \Phi\right) \\
& \times\left[\frac{\Gamma_{0}}{\epsilon_{d}}\left(1+\sum_{\mathbf{q}} A_{\mathbf{q}}^{+} \frac{\omega_{q}^{2}}{\epsilon_{d}^{2}-\omega_{q}^{2}}\right)+\Gamma_{0} \sum_{\mathbf{q}} A_{\mathbf{q}}^{-} \frac{\omega_{q}}{\epsilon_{d}^{2}-\omega_{q}^{2}}\right] .
\end{aligned}
$$

Of particular interest is the point that the magnitude of the interference term can be controlled by coupling the dot to a sonic source. The other factor, $A_{\mathbf{q}}^{-}$, may change sign depending on the relative location of the on-site energy on the dot and the Fermi level, but its magnitude cannot vary much, $-1 \leq 2 n_{d}-1 \leq 1$.

\section{RADIATION EFFECTS ON THE CIRCULATING CURRENT}

The subtle effect that electron-phonon interactions may have on interference-related properties of electrons has been invoked a long time ago by Holstein, ${ }^{73}$ in his theory of the Hall effect in hopping conduction. Holstein proposed that in order to capture the Hall effect, it is necessary to consider processes where the amplitude of the direct electron tunneling between two 'sites' around which the electronic wave functions are localized, interferes with an indirect tunneling amplitude, through an intermediate third site. Moreover, that interference must involve energy-conserving electron transitions to and from the intermediate site, which are assisted by phonons. It turns out that this 'Holstein process' has intriguing consequences for the persistent current in electronically-isolated interferometers. ${ }^{56}$ Since it is of interest to compare the radiation effect on persistent currents in isolated and in open rings, we begin this section with a brief summary of the Holstein process and its consequences for the isolated system, and then analyze the situation in an open ring.

The Holstein mechanism can be explained in a somewhat technical language as follows. Under hopping conduction conditions, transport can be related to transition probabilities. Imagine now the transition probability per unit time, $P_{i j}$, to tunnel from the electronic state localized at $i$ to that localized at $j$. When the system is 
subject to a constant magnetic field, the tunneling amplitude between $i$ and $j$ is multiplied by the magnetic phase acquired from the field along the path $i-j$. Upon taking the absolute value squared of such an amplitude to obtain $P_{i j}$ due to direct hopping alone, the result is independent of the magnetic field. Now let us add to the direct tunneling amplitude between $i$ and $j$ the amplitude for indirect tunneling, for example, the path $i-\ell-j$, where $\ell$ denotes an intermediate site. The transition probability now depends on the total, gauge-invariant, magnetic flux enclosed by the two paths (i.e., the Aharonov-Bohm phase). However, it is an even function of the magnetic phase, as the tunneling amplitudes themselves can always be chosen to be real. As such, this transition probability cannot lead to a dc Hall conduction, which is odd in the field. This line of argument shows that, technically speaking, an imaginary contribution to at least one of the transition amplitudes is required in order to render a term odd in the magnetic phase in the transition probability.

Where can this imaginary part come from? Holstein ${ }^{73}$ argued that when electron-phonon processes are taken into account, the intermediate state becomes in fact a continuum of energy states, consisting of the intermediate electronic energy, and the continuum of phonon energies. This continuum suffices to supply the required imaginary contribution. Roughly speaking, when electron-phonon interactions are accounted for, the tunneling amplitude for the indirect path acquires, for $\epsilon_{\ell}>$ $\epsilon_{i}$, terms such as

$$
\begin{aligned}
J_{i-\ell-j} \sim \sum_{\substack{n_{\mathbf{q}}, \mathbf{q} \\
n_{\mathbf{q}^{\prime}}, \mathbf{q}^{\prime}}} \frac{\left\langle\ell, n_{\mathbf{q}}-1, n_{\mathbf{q}^{\prime}}|V| j, n_{\mathbf{q}}, n_{\mathbf{q}^{\prime}} \pm 1\right\rangle}{\epsilon_{i}-\epsilon_{j} \mp \omega_{q^{\prime}}+i \eta} \\
\times\left\langle j, n_{\mathbf{q}}, n_{\mathbf{q}^{\prime}} \pm 1|V| i, n_{\mathbf{q}}, n_{\mathbf{q}^{\prime}}\right\rangle .
\end{aligned}
$$

Here, $\epsilon_{i}$, etc., denotes electronic site energies, $\eta \rightarrow 0^{+}$, $\omega_{q}$ and $\omega_{q^{\prime}}$ are boson energies, and $n_{\mathbf{q}}$ and $n_{\mathbf{q}^{\prime}}$ are the quantum numbers of the $\mathbf{q}-$ and $\mathbf{q}^{\prime}-$ mode occupations. In Eq. (35), $V$ is the operator that transfers the electron between sites, and at the same time may cause the phonon states to change, obtained after an appropriate ${ }^{73}$ unitary transformation on the electron-boson Hamiltonian, Eq. (22). Since the intermediate state now lies in a continuum of energies, the infinitesimal part $\eta$ leads to a a finite imaginary contribution, provided that the sum of energies in the denominator vanishes, namely, when there is an exact energy conservation, as would be needed to make a real transition ${ }^{74}$ between the initial and intermediate states of the process. We emphasize, however, that the boson created/destroyed in going from $i$ to $j$ is only virtual, exactly the same boson is destroyed/created in going from $j$ to $\ell$. This exact identity is necessary for incoherent phonons in order to retain phase coherence ${ }^{75}$ with the direct process from $i$ to $\ell$. More technically, one uses the relation $1 /(x+i \eta)=\mathcal{P} / x-i \pi \delta(x)$, where $\mathcal{P}$ denotes the principal part. The delta-function term within the infinite sum over the phonon modes gives rise to the required finite imaginary contribution. The resulting imaginary part in $J_{i-\ell-j}$ yields a term odd in the flux in the transition probability. It is worth noting that the energy-conserving process occurs here in the intermediate state of the perturbation theory [of which Eq. (35) is the lowest term] for the combined amplitudes. Recently, this unique process has been proposed as the origin of the anomalous Hall effect in ferromagnetic semiconductors. ${ }^{76}$

The argument above exemplifies the necessity for one resonant process. However, in fact the Holstein process requires at least two resonant electron-phonon processes. This can be explained as follows: The three electronic energies involved in the indirect tunneling and their differences are in general all different. Hence, at least one phonon (the one denoted above by $q$ ) is needed to supply the energy difference $\epsilon_{i}-\epsilon_{\ell}$ between the initial and final electronic states. The second phonon ( $q^{\prime}$ above) appears in the intermediate process, as explained above. We will come back to this point in the following. The phononassisted indirect amplitude, Eq. (35), gives rise also to a contribution which is even in the field (coming from the principal part). That contribution does not require exact energy-conservation within the intermediate state of the perturbation energy (it does however, require the phonon supplying the energy difference between the initial and final electronic states).

The fact that the transition probability per unit time for an electron to hop between two sites may include a term which is odd in the Aharonov-Bohm flux (in addition to the term even in the flux) has an immediate result: detailed balance is broken even at thermal equilibrium. Stated in terms of transition probabilities, $P_{i j}-P_{j i} \neq 0$, and the difference is odd in the magnetic flux. To appreciate the outcome of this observation, let us focus our attention on a triad of three sites, $i, j$, and $\ell$, the smallest cluster in which the doubly-resonant transitions can take place. The transition probability to go from site $i$ to site $j, P_{i j}$, (which includes also the indirect processes via site $\ell$ ), and the transition probability to go from that site to site $\ell$ (now also through the intermediate site $j$ ), $P_{i \ell}$, are such that

$$
P_{i j}+P_{i \ell}=P_{j i}+P_{\ell i}
$$

so that charge balance is maintained at the electronic site $i$. However, since $P_{i j} \neq P_{j i}$, there is a net current circulating around the triad, proportional to $P_{i j}-P_{j i}$, and therefore arising from the Holstein process. That current is additional to the persistent current flowing in this system in the absence of the coupling to the phonon source. In fact, it has been found ${ }^{56}$ that it is always flowing in the reverse direction! (The direction of the current in the triad is determined by delicate effects related to the location of the Fermi level with respect to the site energies, etc.) This current has been therefore termed 'counter-current'. When the full transition probabilities, including the terms even and odd in the magnetic flux, are used in the proper rate equations to find 
the current, the resulting conductivity tensor satisfies the Onsager relations. ${ }^{77}$

Having related the doubly-resonant processes of Holstein to the persistent current, it is worthwhile to reexamine the resonance conditions from the point of view of coherence. As we have pointed above, and as is borne out by the full calculation, ${ }^{56}$ one of the two phonons is common to both interfering tunnelling paths, thus retaining their coherence, ${ }^{75}$ while the other is, as explained above, absorbed and re-emitted by one of the paths, again retaining coherence with the other path. Hence, albeit the fact that the Holstein mechanism also involves a real, energy-conserving, electron-phonon transition, it still contributes in a non-trivial way to the persistent current. However, since this contribution arises from 'real' processes, it requires 'real' phonon modes, namely, non-zero temperatures. One therefore expects that the counter-current will increase with the temperature. On the other hand, the counter-current is also multiplied by the overall damping Debye-Waller factor. Hence, the resulting temperature dependence of the counter-current is non-monotonic. ${ }^{56}$

When the interferometer is connected by leads to external electronic reservoirs, the energy levels on the ring acquire finite widths, given by the imaginary part of the external self-energies. Then, the effect of the coupling to the sonic source is modified. While for discrete states, it required exact energy conservation (up to the width introduced by the coupling to the phonons), here it operates in a finite energy band. Nonetheless, the radiation introduces again a unique effect, which goes beyond that of the Debye-Waller exponent. In the present situation the sonic effect is of a lower order in the electron-phonon coupling, and may exist even in the $T \rightarrow 0$ limit as will be discussed later. (For a concise summary of this result see Ref. 68.)

Indeed, inserting the expansion of the dot Green function at small electron-phonon coupling, Eq. (25), into our general result for the circulating current, Eq. (8), yields

$$
I_{p c}=I_{p c}^{0}+\Delta I_{p c}
$$

where $I_{p c}^{0}$ is the persistent current of the non-interacting interferometer, given by Eq. (20) above, and $\Delta I_{p c}$ is the acousto-persistent current, given, within our approximation, by

$$
\begin{aligned}
& \Delta I_{p c}=\int \frac{d \omega}{\pi} \frac{f_{\ell}(\omega)+f_{r}(\omega)}{4} \\
& \times \sum_{\mathbf{q}}\left[A_{q}^{-} \frac{\partial}{\partial \Phi}\left(\delta^{0}\left(\omega+\omega_{q}\right)-\delta^{0}\left(\omega-\omega_{q}\right)\right)\right. \\
& \left.+A_{q}^{+} \frac{\partial}{\partial \Phi}\left(\delta^{0}\left(\omega+\omega_{q}\right)+\delta^{0}\left(\omega-\omega_{q}\right)-2 \delta^{0}(\omega)\right)\right],
\end{aligned}
$$

where $\delta^{0}$, the Friedel phase of the non interacting system, is given in Eq. (21), and $A_{\mathbf{q}}^{ \pm}$is defined in Eqs.
(33). The acousto-induced persistent current, $\Delta I_{p c}$, consists of two parts: The first term depends only on the dot's occupation, $n_{d}$, and its sign may change according to the relative location of $\epsilon_{d}$ with respect to the Fermi energy. The second term in Eq. (38) is dominated by the phonon occupations [see Eq. (33)], via $A_{\mathbf{q}}^{+}$. [Note that the term $-2 \delta^{0}(\omega)$ there comes from the expansion of the Debye-Waller exponent.] Examining this contribution shows that by shining a beam of phonons of a specific frequency, the magnitude of that term can be enhanced and controlled experimentally, as long as the temperature of the electronic system and the intensity of the phonon source $N_{q}$ are low enough to retain coherent motion of the electrons. (The intensity is also limited in the present calculation by the assumption of weak electron-phonon coupling; However, there is no conceptual difficulty to extend the calculation to stronger values.) Similar considerations apply to photons. Both the precise magnitude of these effects and the above bounds depend on the detailed geometry of the dot and on the acoustic (or electromagnetic) mismatch.

It is important to appreciate the difference between this result and the corresponding one found for the isolated ring. In the isolated ring, the Holstein process ${ }^{55}$ required the emission (absorption) of a specific phonon, with the exact excitation energy of the electron on the ring. In the present case, the coupling to the leads turns the bound state into a resonance, with a width $\Gamma_{0}$ which vanishes when the ring is decoupled from the leads. As a result, there is always some overlap between the tail of the Green function $G_{d d}^{R 0}(\omega)$ and the Fermi distribution $f(\omega)$, yielding contributions from Holstein-like processes via phonons with many (including very low) energies. Indeed, each contribution to $\Delta I_{p c}$ contains the phase $\delta^{0}(\omega)$, which vanishes with $\Gamma_{0}\left(\delta^{0} \sim \Gamma_{0} /\left|\epsilon_{d}\right|\right.$ far from the resonance). In particular, this results in a nonzero $\Delta I_{p c}$ even at zero temperature: In that limit, if $\epsilon_{d}<\mu_{\ell}=\mu_{r}=0$, then $n_{d}=1$. Even with no phonons, $N_{q}=0$, the square brackets in Eq. (38) become proportional to $\partial\left[\delta^{0}\left(\omega+\omega_{q}\right)-\delta^{0}(\omega)\right] / \partial \Phi$, reflecting processes which begin by an emission of phonons. None of this remains for the isolated ring, when $\Gamma_{0}=0$.

To obtain explicit expressions, we now evaluate the frequency integration appearing in Eq. (38). Since we are operating within the linear response regime, the voltage is not essential to our effect and we may safely write in Eq. (38) $f_{\ell}(\omega)=f_{r}(\omega) \equiv f(\omega)$. Furthermore, we take the electronic temperature to be low compared to all other energies, so that $f(\omega) \approx \Theta(-\omega)$. We also take the typical phonon frequency to be much smaller than the large band-width in the leads. With these approximations the frequency-integration in Eq. (38) is easily performed, to yield

$$
\begin{aligned}
\Delta I_{p c} & =\frac{\Gamma_{0}}{4 \pi} \sin \Phi \sum_{\mathbf{q}}\left[A_{\mathbf{q}}^{+}\left(F\left(\omega_{q}\right)+F\left(-\omega_{q}\right)-2 F(0)\right)\right. \\
& \left.+A_{\mathbf{q}}^{-}\left(F\left(\omega_{q}\right)-F\left(-\omega_{q}\right)\right)\right]
\end{aligned}
$$


where $F(\omega)$ is given in terms of $\delta^{0}(\omega)$, Eq. (21),

$$
F(\omega)=-\sqrt{T_{B} R_{B}} \delta^{0}(\omega)-T_{B} \ln \left|\sin \delta^{0}(\omega)\right| .
$$

Note again that the dependence of the acousto-persistent current on the phonon frequency is determined by the Friedel phase of the dot at that frequency. To leading order in the strength of the electron-phonon coupling, the magnitude of the first term in $\Delta I_{p c}$ is proportional to $A_{\mathbf{q}}^{+}$, and thus grows linearly with the occupation number of the acoustic modes acting on the dot, $N_{q}$. In fact, the acousto-persistent current contains two types of contributions: the part associated with $F(0)$, which simply represents the 'trivial' Debye-Waller renormalization of the current, and the novel frequency-dependent parts, which reflect the change in the persistent current due to Holstein-like processes.

The above discussion holds at thermal equilibrium. There, the electron-phonon interaction does not enhance the persistent current. On the other hand, taking the phonon source out of equilibrium at a certain frequency range may lead to enhancement. On a speculative level, remembering that the Debye-Waller exponent depends on the sum of all phonon occupations (weighted by their couplings to the electronic levels), while the countercurrent depends only on the phonon occupations of the two resonating frequencies, one may visualize the following set-up: Suppose that one shines on the electronic system a high intensity beam of non-equilibrium phonons (or photons) with a narrow frequency range around, say, $\omega_{0}$. The counter-current, resulting from resonant transitions, will be significantly affected by the non-equilibrium phonons only when $\omega_{0}$ will be close to the differences $\left|\epsilon_{i}-\epsilon_{j}\right|$ or $\left|\epsilon_{i}-\epsilon_{\ell}\right|$. The effect on the Debye-Waller factor, on the other hand, will be small for a narrowband beam. In this way, the counter-current will initially increase with the intensity of this radiation, until the Debye-Waller/decoherence/heating effects will take over and the entire persistent current will disappear.

We have not emphasized in this paper the contribution of non-Holstein processes [i.e., those arising from the principal part in Eq. (35)]. Such processes are not specific to a definite phonon frequency, and therefore can not be increased without heating/decohering the system.

\section{SUMMARY}

We have considered the effect of coupling the electrons to a boson source on their interference pattern in an Aharonov-Bohm interferometer, and in particular focused our attention on the modifications in the transport current and in the circulating current. In both cases, there appears the overall Debye-Waller exponent, which reduces the interference term (as well as the 'classical' term), and hence the currents, as the temperature is raised. All boson modes contribute to the Debye-Waller factor. This outcome of the coupling to the boson source is not surprising. However, in both cases, there is an additional contribution, which is confined to a bounded range of phonon energies, dictated by the electronic energies.

In the case of hopping conduction, which involves transitions between discrete localized electronic states that in general differ in energy, a phonon (common to the two paths) is necessary to conserve energy in the overall hopping process. ${ }^{74}$ In the case of an open interferometer, that phonon is not necessary, since the electronic states on the two leads form continua and overlap in energy. To obtain a term odd in the magnetic field in the hopping regime, another, 'second', phonon is needed, which has to conserve the total energy between the initial and intermediate states. ${ }^{73}$ The reverse phonon process (namely, restoring the phonon system back to its original state) then occurs between the intermediate and final states, thus retaining phase memory in the overall process (which can then interfere with another phonon-less path). The conservation of energy in the intermediate state is a rather unusual feature, which introduces an imaginary part to the hopping amplitude for that path, and hence a nontrivial phase. That phase was crucial for the theory of the Hall effect in the hopping regime. Here, the process appears at a lower order in the electron-phonon interaction, as compared to the situation in isolated rings with localized electronic states. ${ }^{56}$ In addition, the intermediate electronic state acquires a width via coupling to the leads. Therefore the process may exist even at zero temperature. This is due to the finite overlap of the intermediate electronic state with the "band".

Because this novel contribution to the currents comes from a confined range of boson frequencies, it is expected that by modulating the intensity of the radiation in that frequency range, it will be possible to manipulate the magnitude of the currents. This will require boson intensities low enough to retain the coherent motion of the electrons. However, the fact that this unique effect is confined to a rather narrow region of boson frequencies (while the detrimental Debye-Waller factor comprises all boson frequencies) gives some hope that such an acoustomagnetic effect is feasible in experiments.

\section{ACKNOWLEDGMENTS}

This project was carried out in a center of excellence supported by the Israel Science Foundation. It was also partially supported by the German-Israeli Foundation (GIF), by the U.S. Department of energy, Office of Science, through contact No. W-31-109-ENG-38, and by the German Federal Ministry of Education and Research (BMBF), within the framework of the German Israeli Project Cooperation (DIP). 


\section{APPENDIX A: DETAILS OF THE CURRENT CALCULATION}

As is clearly explained in Ref. 62 (see also Ref. 61), the Green functions required in the Keldysh technique can be found by considering the time-ordered Green functions, $G^{T}$. The latter satisfy the frequency-dependent Dyson equation for $G^{T}(\omega)$,

$$
G^{T}=G^{0 T}+G^{0 T} \Sigma^{T} G^{T} .
$$

Then, the retarded $\left(G^{R}\right)$ and the advanced $\left(G^{A}\right)$ Green functions are obtained by replacing $T$ above by $R$ or $A$, while $G^{<}$is found according to the rule ${ }^{62}$

$$
(\Sigma G)^{<}=\Sigma^{R} G^{<}+\Sigma^{<} G^{A},
$$

and similarly for any other product. In the following, we omit for brevity the notation $T$ from the time-ordered Green functions.

\section{The calculation of the partial currents}

We now apply the Keldysh method to calculate the partial currents, defined in Eq. (9). Our aim is to express these currents in terms of the dot Green function, $G_{d d}$.

We begin with the calculation of $I_{1}$. The required Green functions $G_{k d}^{<}$and $G_{d k}^{<}$are obtained as follows. The equations-of-motion for the temporal Fourier transforms of the time-ordered counterparts read

$$
\begin{aligned}
& \left(\omega-\epsilon_{k}\right) G_{k d}=V_{k} G_{d d}+v_{k} G_{0 d}, \\
& G_{d k}\left(\omega-\epsilon_{k}\right)=V_{k}^{*} G_{d d}+v_{k}^{*} G_{d 0} .
\end{aligned}
$$

In order to use the rule (A2), we re-write these two equations in the form

$$
\begin{aligned}
G_{k d} & =V_{k} g_{k} G_{d d}+v_{k} g_{k} G_{0 d}, \\
G_{d k} & =V_{k}^{*} G_{d d} g_{k}+v_{k}^{*} G_{d 0} g_{k},
\end{aligned}
$$

in which $g_{k}$ is the free Green function of the left lead, namely

$$
g_{k}^{R, A}=\frac{1}{\omega \pm i \eta-\epsilon_{k}}, \quad g_{k}^{<}=f_{\ell}(\omega)\left(g_{k}^{A}-g_{k}^{R}\right) .
$$

Here $\eta \rightarrow 0^{+}$, and

$$
f_{\ell}=\frac{1}{e^{\beta\left(\omega-\mu_{\ell}\right)}+1}
$$

is the electron distribution in the left electronic reservoir, and Eq. (10) has been employed to obtain $g_{k}^{<}$. Since we assume that the two leads in Fig. 1 are identical except for being connected to reservoirs of different chemical potentials, the free Green functions of the right lead are given by Eqs. (A5), with $f_{\ell}$ replaced by $f_{r}$. For brevity, the dependence on the frequency $\omega$ will be suppressed in most of the equations.

Using now the rule (A2), we find

$$
\begin{aligned}
& G_{k d}^{<}=V_{k}\left(g_{k}^{R} G_{d d}^{<}+g_{k}^{<} G_{d d}^{A}\right)+v_{k}\left(g_{k}^{R} G_{0 d}^{<}+g_{k}^{<} G_{0 d}^{A}\right), \\
& G_{d k}^{<}=V_{k}^{*}\left(G_{d d}^{<} g_{k}^{A}+G_{d d}^{R} g_{k}^{<}\right)+v_{k}^{*}\left(G_{d 0}^{<} g_{k}^{A}+G_{d 0}^{R} g_{k}^{<}\right) .
\end{aligned}
$$

Inserting Eqs. (A7) into $I_{1}$, Eq. (9), and writing explicitly the couplings $V_{k}$ and $v_{k}$ from Eqs. (5), one finds

$$
\begin{aligned}
I_{1} & =e \int \frac{d \omega}{2 \pi} \frac{2}{N} \sum_{k} \sin ^{2} k\left[j _ { \ell } ^ { 2 } \left(G_{d d}^{<}\left(g_{k}^{R}-g_{k}^{A}\right)\right.\right. \\
& \left.+g_{k}^{<}\left(G_{d d}^{A}-G_{d d}^{R}\right)\right)+j_{\ell} i_{\ell} e^{i \phi_{\ell}}\left(g_{k}^{R} G_{0 d}^{<}+g_{k}^{<} G_{0 d}^{A}\right) \\
& \left.-j_{\ell} i_{\ell} e^{-i \phi_{\ell}}\left(g_{k}^{<} G_{d 0}^{R}+g_{k}^{A} G_{d 0}^{<}\right)\right] .
\end{aligned}
$$

When the explicit expressions for $g_{k}^{A}, g_{k}^{R}$, and $g_{k}^{<}$[see Eqs. (A5)] are inserted into Eq. (A8), it turns out that it is useful to define

$$
\alpha^{R, A}=\frac{2}{N} \sum_{k} g_{k}^{R, A} \sin ^{2} k,
$$

and

$$
\Delta=\alpha^{A}-\alpha^{R} \equiv \frac{4 \pi i}{N} \sum_{k} \delta\left(\omega-\epsilon_{k}\right) \sin ^{2} k .
$$

With these notations, the partial current $I_{1}$ becomes

$$
\begin{aligned}
I_{1} & =e \int \frac{d \omega}{2 \pi}\left(-\Delta j_{\ell}^{2}\left[G_{d d}^{<}+f_{\ell}\left(G_{d d}^{R}-G_{d d}^{A}\right)\right]\right. \\
& +j_{\ell} i_{\ell} e^{i \phi_{\ell}}\left[f_{\ell} \Delta G_{0 d}^{A}+\alpha^{R} G_{0 d}^{<}\right] \\
& \left.-j_{\ell} i_{\ell} e^{-i \phi_{\ell}}\left[\alpha^{A} G_{d 0}^{<}+f_{\ell} \Delta G_{d 0}^{R}\right]\right) .
\end{aligned}
$$

The next step is the find the Green functions $G_{0 d}$ and $G_{d 0}$ in terms of the dot Green function $G_{d d}$. This is accomplished as follows. The equation-of-motion for the time-ordered Green function $G_{0 d}$ reads

$$
G_{0 d}=g_{0}\left(\sum_{k} v_{k}^{*} G_{k d}+\{k \rightarrow p\}\right),
$$

in which the notations $\{k \rightarrow p\}$ stand for the analogous sum on the right lead, and $g_{0}$ is the free Green function on the reference site, with

$$
g_{0}^{R, A}=\frac{1}{\omega \pm i \eta-\epsilon_{0}} .
$$

Since the bare reference site is not coupled to any electronic reservoir, the free Keldysh Green function for that site vanishes,

$$
g_{0}^{<}=0
$$


Making use of Eqs. (A4) we have

$$
G_{0 d}^{A}=g_{0}^{A}\left[\sum_{k} v_{k}^{*} g_{k}^{A}\left(V_{k} G_{d d}^{A}+v_{k} G_{0 d}^{A}\right)+\{k \rightarrow p\}\right],
$$

which yields

$$
G_{0 d}^{A}=Y D_{0}^{A} \alpha^{A} G_{d d}^{A}
$$

where $D_{0}^{A}$ is the reference site Green function when the upper arm of the ring is cut off,

$$
D_{0}^{A}=\frac{1}{\omega-i \eta-\epsilon_{0}-\alpha^{A}\left(i_{\ell}^{2}+i_{r}^{2}\right)},
$$

and $Y$ denotes the interference coupling,

$$
Y=i_{\ell} j_{\ell} e^{-i \phi_{\ell}}+i_{r} j_{r} e^{i \phi_{r}} .
$$

The retarded Green function $G_{0 d}^{R}$ is obtained from Eq. (A16) by interchanging ' $A$ ' into ' $R$ '. A similar calculation yields

$$
G_{d 0}^{A}=Y^{*} D_{0}^{A} \alpha^{A} G_{d d}^{A} .
$$

[We remind the reader that the two leads are assumed to be identical except that they are connected to electronic reservoirs with different chemical potentials.] Turning now to the calculation of $G_{0 d}^{<}$and $G_{d 0}^{<}$, we first apply the rule (A2) to Eq. (A12), to obtain

$$
\begin{aligned}
G_{0 d}^{<} & =g_{0}^{R}\left[\sum _ { k } v _ { k } ^ { * } \left(V_{k}\left(g_{k}^{R} G_{d d}^{<}+g_{k}^{<} G_{d d}^{A}\right)\right.\right. \\
& \left.\left.+v_{k}\left(g_{k}^{R} G_{0 d}^{<}+g_{k}^{<} G_{0 d}^{A}\right)\right)+\{k \rightarrow p\}\right] .
\end{aligned}
$$

Then we collect the coefficients of $G_{0 d}^{<}$[using Eq. (A17)] to find

$$
\begin{aligned}
G_{0 d}^{<} & =D_{0}^{R}\left[\sum _ { k } v _ { k } ^ { * } \left(V_{k}\left(g_{k}^{R} G_{d d}^{<}+g_{k}^{<} G_{d d}^{A}\right)\right.\right. \\
& \left.\left.+v_{k} g_{k}^{<} G_{0 d}^{A}\right)+\{k \rightarrow p\}\right] .
\end{aligned}
$$

Finally we insert here Eq. (A16) to obtain

$$
\begin{aligned}
G_{0 d}^{<} & =\alpha^{R} D_{0}^{R} Y\left(G_{d d}^{<}-f_{\ell} G_{d d}^{A}\right)+f_{\ell} \alpha^{A} D_{0}^{A} Y G_{d d}^{A} \\
& +\Delta D_{0}^{R} G_{d d}^{A}\left(f_{r}-f_{\ell}\right) i_{r}\left(J_{r}^{R}(\Phi)\right)^{*} e^{i \phi_{r}}
\end{aligned}
$$

A similar calculation yields

$$
\begin{aligned}
G_{d 0}^{<} & =\alpha^{A} D_{0}^{A} Y^{*}\left(G_{d d}^{<}+f_{\ell} G_{d d}^{R}\right)-f_{\ell} \alpha^{R} D_{0}^{R} Y^{*} G_{d d}^{R} \\
& +\Delta D_{0}^{A} G_{d d}^{R}\left(f_{r}-f_{\ell}\right) i_{r} J_{r}^{R}(\Phi) e^{-i \phi_{r}} .
\end{aligned}
$$

Here we have introduced the effective couplings connecting the quantum dot to the right part of the ring,

$$
J_{r}^{R}(\Phi)=j_{r}+i_{r} \alpha^{R} D_{0}^{R}\left(i_{\ell} j_{\ell} e^{i \Phi}+i_{r} j_{r}\right),
$$

and to the left side,

$$
J_{\ell}^{R}(\Phi)=j_{\ell}+i_{\ell} \alpha^{R} D_{0}^{R}\left(i_{\ell} j_{\ell}+i_{r} j_{r} e^{-i \Phi}\right),
$$

and used the relation

$$
D_{0}^{R}-D_{0}^{A}=-\Delta D_{0}^{R} D_{0}^{A}\left(i_{\ell}^{2}+i_{r}^{2}\right) .
$$

Introducing all these results into $I_{1}$, Eq. (A11), gives that partial current in terms of the dot Green function,

$$
\begin{aligned}
& I_{1}=e \int \frac{d \omega}{2 \pi}\left\{2 i \sin \Phi\left(i_{\ell} j_{\ell} i_{r} j_{r}\right)\left[\left(\alpha^{A}\right)^{2} D_{0}^{A} G_{d d}^{A}-c c\right] f_{\ell}\right. \\
+ & {\left[\alpha^{R} j_{\ell} J_{\ell}^{R}(-\Phi)-c c\right]\left[G_{d d}^{<}+f_{\ell}\left(G_{d d}^{R}-G_{d d}^{A}\right)\right] } \\
+ & {\left.\left[\left(J_{r}^{R}(\Phi)\right)^{*} j_{\ell} i_{\ell} i_{r} e^{i \Phi} \alpha^{R} D_{0}^{R} G_{d d}^{A}-c c\right] \Delta\left(f_{r}-f_{\ell}\right)\right\} . }
\end{aligned}
$$

[Note that $\Delta^{*}=-\Delta$.] Examining the expression for the partial current $I_{3}$, Eq. (9), it is seen that it is obtained from $I_{1}$, upon the replacements $\ell \leftrightarrow r$ with $k \leftrightarrow p$, and $\phi_{\ell} \leftrightarrow-\phi_{r}$, namely $\Phi \rightarrow-\Phi$. Then [see Eqs. (A24) and (A25)] $J_{\ell}^{R}(-\Phi) \leftrightarrow J_{r}^{R}(\Phi)$.

Next we consider the partial current $I_{2}$. A similar calculation to the one leading to Eq. (A11) yields

$$
\begin{aligned}
I_{2} & =e \int \frac{d \omega}{2 \pi}\left(-\Delta i_{\ell}^{2}\left[G_{00}^{<}+f_{\ell}\left(G_{00}^{R}-G_{00}^{A}\right)\right]\right. \\
& +j_{\ell} i_{\ell} e^{-i \phi_{\ell}}\left[\alpha^{R} G_{d 0}^{<}+\Delta f_{\ell} G_{d 0}^{A}\right] \\
& \left.-j_{\ell} i_{\ell} e^{i \phi_{\ell}}\left[\Delta f_{\ell} G_{0 d}^{R}+\alpha^{A} G_{0 d}^{<}\right]\right) .
\end{aligned}
$$

In order to express this current in terms of the dot Green function, we need to calculate the reference site Green function $G_{00}$. The equation-of-motion for the time-ordered counterpart gives

$$
G_{00}=g_{0}+g_{0}\left[\sum_{k} v_{k}^{*} G_{k 0}+\{k \leftrightarrow p\}\right],
$$

with

$$
G_{k 0}=V_{k} g_{k} G_{d 0}+v_{k} g_{k} G_{00} .
$$

Making use of Eqs. (A17) and (A19), we find

$$
G_{00}^{R}=D_{0}^{R}+\left(D_{0}^{R} \alpha^{R}\right)^{2}|Y|^{2} G_{d d}^{R} .
$$

The advanced function $G_{00}^{A}$ is given by this equation upon interchanging ' $R$ ' with ' $A$ '. Next we apply the rule (A2) to Eqs. (A29) and (A30) to obtain

$$
\begin{aligned}
G_{00}^{<} & =D_{0}^{R}\left[Y \alpha^{R} G_{d 0}^{<}+\Delta\left(i_{\ell}^{2} f_{\ell}+i_{r}^{2} f_{r}\right) G_{00}^{A}\right. \\
& \left.+\Delta\left(i_{\ell} j_{\ell} e^{-i \phi_{\ell}} f_{\ell}+i_{r} j_{r} e^{i \phi_{r}} f_{r}\right) G_{d 0}^{A}\right] .
\end{aligned}
$$

It remains to insert here Eqs. (A19), (A23), and (A31), to obtain 


$$
\begin{aligned}
& G_{00}^{<}=\Delta D_{0}^{R} D_{0}^{A}\left(i_{\ell}^{2} f_{\ell}+i_{r}^{2} f_{r}\right) \\
+ & \left|Y \alpha^{R} D_{0}^{R}\right|^{2}\left[G_{d d}^{<}+f_{\ell}\left(G_{d d}^{R}-G_{d d}^{A}\right)\right] \\
+ & {\left[\Delta \alpha^{R} D_{0}^{R} D_{0}^{A} Y i_{r} J_{r}^{R}(\Phi) e^{-i \phi_{r}}\left(f_{r}-f_{\ell}\right) G_{d d}^{R}-c c\right] } \\
+ & |Y|^{2} f_{\ell}\left[\left(\alpha^{A} D_{0}^{A}\right)^{2} G_{d d}^{A}-c c\right] .
\end{aligned}
$$

The first term here is the contribution of the lower arm of the ring alone; The other terms arise from interference.

Introducing these results into Eq. (A28) for $I_{2}$, we find

$$
\begin{aligned}
I_{2}=e \int \frac{d \omega}{2 \pi}\left\{D_{0}^{R} D_{0}^{A} i_{\ell}^{2} i_{r}^{2} \Delta^{2}\left(f_{\ell}-f_{r}\right)\right. \\
-2 i \sin \Phi\left(i_{\ell} j_{\ell} i_{r} j_{r}\right)\left[\left(\alpha^{A}\right)^{2} D_{0}^{A} G_{d d}^{A}-c c\right] f_{\ell} \\
+\left[\alpha^{R} J_{\ell}^{R}(-\Phi)\left(\left(J_{\ell}^{R}(-\Phi)\right)^{*}-j_{\ell}\right)-c c\right] \\
\quad \times\left[G_{d d}^{<}+f_{\ell}\left(G_{d d}^{R}-G_{d d}^{A}\right)\right] \\
+\left[\alpha^{R} D_{0}^{A} i_{\ell} i_{r} e^{-i \Phi} G_{d d}^{R} J_{r}^{R}(\Phi)\right. \\
\left.\left.\quad \times\left(j_{\ell}-\Delta D_{0}^{R} i_{\ell} e^{i \phi_{\ell}} Y\right)-c c\right] \Delta\left(f_{r}-f_{\ell}\right)\right\} .
\end{aligned}
$$

Examining the expression for the partial current $I_{4}$, Eq. (9), it is seen that it is obtained from $I_{2}$, upon the replacements $\ell \leftrightarrow r$ and $\phi_{\ell} \leftrightarrow-\phi_{r}$.

\section{Current conservation}

Having obtained the partial currents in terms of the dot Green functions, we now examine the consequences. The important point to bear in mind is that in the presence of interactions (confined to the quantum dot alone), those Green functions are not known, and may be found only approximately. Therefore, imposing current conservation will yield general relations which the $G_{d d}$ 's have to satisfy.

Current conservation means (see Fig. 1) that $I_{1}+I_{3}=$ 0 , and $I_{2}+I_{4}=0$. A lengthy calculation of the sum $I_{2}+I_{4}$, using Eq. (A34), shows that it indeed vanishes. In contrast, the sum of the currents on the interferometer arm containing the dot, using Eq. (A27), gives

$$
\begin{aligned}
& I_{1}+I_{3}=e \int \frac{d \omega}{2 \pi} \\
& \times\left[\left(\Sigma_{\mathrm{ext}}^{R}-\Sigma_{\mathrm{ext}}^{A}\right) G_{d d}^{<}+\Sigma_{\mathrm{ext}}^{<}\left(G_{d d}^{A}-G_{d d}^{R}\right)\right],
\end{aligned}
$$

in which $\Sigma_{\text {ext }}$ denotes the self-energy of the dot Green function, which arises from the connection of the dot to the interferometer and the leads. This relation may be verified as follows. The external self-energy, $\Sigma_{\text {ext }}$, is found from the Dyson equation (A1), using only the noninteracting parts of the Hamiltonian, Eq. (1), and Eq. (A4),

$$
\Sigma_{\mathrm{ext}} G_{d d}=\left[\sum_{k} V_{k} g_{k}\left(V_{k} G_{d d}+v_{k} G_{0 d}\right)+\{k \rightarrow p\}\right]
$$

Employing Eqs. (A16) and (A22), together with the rule (A2), one then finds

$$
\Sigma_{\mathrm{ext}}^{R}=\alpha^{R}\left(j_{\ell}^{2}+j_{r}^{2}+\alpha^{R} D_{0}^{R}|Y|^{2}\right)
$$

with an analogous expression for $\Sigma_{\text {ext }}^{A}$, and

$$
\Sigma_{\mathrm{ext}}^{<}=\Delta\left[f_{\ell}\left|J_{\ell}^{R}(\Phi)\right|^{2}+f_{r}\left|J_{r}^{R}(\Phi)\right|^{2}\right] .
$$

When one combines $I_{1}$ and $I_{3}$, and uses the results Eqs. (A37) and (A38), one arrives at Eq. (A35). Note that, using Eqs. (A24), (A25) and (A37), one has

$$
\begin{aligned}
\Sigma_{\mathrm{ext}}^{A}-\Sigma_{\mathrm{ext}}^{R} & =\Delta\left[\left|J_{\ell}^{R}(\Phi)\right|^{2}+\left|J_{r}^{R}(\Phi)\right|^{2}\right] \\
& =\Delta\left[\left|J_{\ell}^{R}(-\Phi)\right|^{2}+\left|J_{r}^{R}(-\Phi)\right|^{2}\right] .
\end{aligned}
$$

When the electronic system is un-biased, namely, when the chemical potentials on both reservoirs are identical, we have

$$
f_{\ell}=f_{r} \equiv f_{t h}
$$

Then Eqs. (A38) and (A39) give

$$
\Sigma_{\text {ext }}^{<}=f_{t h}\left(\Sigma_{\text {ext }}^{A}-\Sigma_{\text {ext }}^{R}\right) .
$$

Without the bias, one also has ${ }^{62}$

$$
G_{d d}^{<}=f_{t h}\left(G_{d d}^{A}-G_{d d}^{R}\right) .
$$

It follows that without a bias, the integrand in Eq. (A35) vanishes. In other words, when the ring is not biased, current conservation is trivially satisfied.

Another case in which Eq. (A35) is trivially satisfied is when the dot is free of any interactions. Then the dot Green function (marked by the superscript '0') obeys

$$
\begin{aligned}
G_{d d}^{R 0} & =\frac{1}{\omega-\epsilon_{d}-\Sigma_{\mathrm{ext}}^{R}}, \\
G_{d d}^{R 0}-G_{d d}^{A 0} & =G_{d d}^{A 0}\left[\Sigma_{\mathrm{ext}}^{R}-\Sigma_{\mathrm{ext}}^{A}\right] G_{d d}^{R 0},
\end{aligned}
$$

where for simplicity it has been assumed that there is only a single electronic level on the dot, denoted $\epsilon_{d}$. For the non-interacting system one also has ${ }^{62}$

$$
G_{d d}^{<0}=G_{d d}^{A 0} \Sigma_{\mathrm{ext}}^{<} G_{d d}^{R 0},
$$

and therefore, again, the integrand in Eq. (A35) vanishes.

Had we known the exact forms of $G_{d d}^{R, A}$ and $G_{d d}^{<}$for the interacting electronic system, we would have found that current conservation is also satisfied when the ring is biased. However, as mentioned above, the dot Green 
function is not known exactly. Therefore, we may regard the relation Eq. (A35) as a condition imposed on $G_{d d}^{R, A}$ and $G_{d d}^{<}$. In order to make a practical use of this condition, we assume that the main contribution to the $\omega$-integration in Eq. (A35) comes from frequencies at about the Fermi level of the electrons (remembering that the ring is only slightly biased), and therefore we may write

$$
G_{d d}^{<}=\Sigma_{\mathrm{ext}}^{<} \frac{G_{d d}^{R}-G_{d d}^{A}}{\Sigma_{\mathrm{ext}}^{R}-\Sigma_{\mathrm{ext}}^{A}} .
$$

This approximation is insufficient to determine the dot Green function, but at least it eliminates the necessity to calculate the Keldysh Green function $G_{d d}^{<}$, and ensures that the current through the ring is conserved. Without a bias voltage, Eq. (A45) holds exactly, yielding in particular the charge in the dot, which is equal to the expectation value of the dot occupation, $n_{d}=$ $-i \int(d \omega / 2 \pi) G_{d d}^{<}(\omega)$, [see Eq. (10)]. With a finite bias, Eq. (A45) is only approximate. However, the implied dependence of $n_{d}$ on the bias voltage will still obey current conservation. The approximation leading to Eq. (A45) is sometimes referred to as the 'wide-band' approximation. ${ }^{61}$

With the approximation (A45) and Eqs. (A38) and (A39), one has

$$
\begin{aligned}
G_{d d}^{<}+f_{\ell}\left(G_{d d}^{R}-G_{d d}^{A}\right) & =\frac{G_{d d}^{R}-G_{d d}^{A}}{\Sigma_{\mathrm{ext}}^{R}-\Sigma_{\mathrm{ext}}^{A}} \Delta\left|J_{r}^{R}(\Phi)\right|^{2}\left(f_{r}-f_{\ell}\right), \\
G_{d d}^{<}+f_{r}\left(G_{d d}^{R}-G_{d d}^{A}\right) & =\frac{G_{d d}^{R}-G_{d d}^{A}}{\Sigma_{\mathrm{ext}}^{R}-\Sigma_{\mathrm{ext}}^{A}} \Delta\left|J_{\ell}^{R}(\Phi)\right|^{2}\left(f_{\ell}-f_{r}\right) .
\end{aligned}
$$

We emphasize again that when the ring is not biased, or when the dot is free of any interactions, the relations (A45) and (A46) are always satisfied.

\section{The current through the ring}

A glance at Fig. 1 shows that the current through the ring, $I$, is given by $I=I_{1}+I_{2}=-I_{3}-I_{4}$. This current is conveniently found from Eqs. (A27) and (A34) by calculating $\left(I_{1}+I_{2}-I_{3}-I_{4}\right) / 2$. The terms proportional to $\sin \Phi$ are then cancelled, and one is left with

$$
\begin{aligned}
I= & e \int \frac{d \omega}{2 \pi}\left\{-\frac{\Delta}{2}\left|J_{\ell}^{R}(-\Phi)\right|^{2}\left[G_{d d}^{<}+f_{\ell}\left(G_{d d}^{R}-G_{d d}^{A}\right)\right]\right. \\
+ & \frac{\Delta}{2}\left|J_{r}^{R}(-\Phi)\right|^{2}\left[G_{d d}^{<}+f_{r}\left(G_{d d}^{R}-G_{d d}^{A}\right)\right] \\
+ & \left(f_{\ell}-f_{r}\right) \Delta^{2} i_{\ell}^{2} i_{r}^{2}\left|D_{0}^{R}\right|^{2}\left[1+G_{d d}^{R} \Sigma_{\mathrm{ext}}^{R}+G_{d d}^{A} \Sigma_{\mathrm{ext}}^{A}\right] \\
+ & \left(f_{\ell}-f_{r}\right) \Delta^{2} i_{\ell} i_{r} j_{\ell} j_{r} \cos \Phi \\
& \times D_{0}^{R}\left[\left(1+\alpha^{A} D_{0}^{A}\left(i_{\ell}^{2}+i_{r}^{2}\right)\right]\left(G_{d d}^{R}+G_{d d}^{A}\right)\right\} .
\end{aligned}
$$

[Note that the quantity $D_{0}^{R}\left[1+\alpha^{A} D_{0}^{A}\left(i_{\ell}^{2}+i_{r}^{2}\right)\right]$ is real, see Eq. (A17).] Making use of the approximation (A45) and the resulting relations (A46), the current through the ring takes the form

$$
I=I_{\text {ref }}+I_{\text {dot }}+I_{\text {int }},
$$

where the first term here, $I_{\text {ref }}$, reduces to the current through the reference arm when the other arm is disconnected,

$$
\begin{gathered}
I_{\mathrm{ref}}=e \int \frac{d \omega}{2 \pi}\left(f_{\ell}-f_{r}\right) \Delta^{2} i_{\ell}^{2} i_{r}^{2}\left|D_{0}^{R}\right|^{2}\left(1+G_{d d}^{R} \Sigma_{\mathrm{ext}}^{R}\right. \\
\left.+G_{d d}^{A} \Sigma_{\mathrm{ext}}^{A}+\Sigma_{\mathrm{ext}}^{R} \Sigma_{\mathrm{ext}}^{A} \frac{G_{d d}^{R}-G_{d d}^{A}}{\Sigma_{\mathrm{ext}}^{R}-\Sigma_{\mathrm{ext}}^{A}}\right) .
\end{gathered}
$$

Similarly, the current $I_{\mathrm{dot}}$, which reduces to the one flowing in the absence of the reference arm, is

$$
\begin{aligned}
I_{\mathrm{dot}}= & e \int \frac{d \omega}{2 \pi}\left(f_{\ell}-f_{r}\right) \Delta^{2} j_{\ell}^{2} j_{r}^{2} \\
& \times\left|1+\alpha^{R} D_{0}^{R}\left(i_{\ell}^{2}+i_{r}^{2}\right)\right|^{2} \frac{G_{d d}^{R}-G_{d d}^{A}}{\Sigma_{\mathrm{ext}}^{R}-\Sigma_{\mathrm{ext}}^{A}} .
\end{aligned}
$$

Each of these currents is 'dressed' by processes in which the electrons travel through the other branch. As might be expected, the interference between the two branches always appears via the product $j_{\ell} j_{r} i_{\ell} i_{r} \cos \Phi$. In addition to appearing implicitly, via $\Sigma_{\text {ext }}$, in $I_{\text {dot }}$ and $I_{\text {ref }}$, this product appears explicitly in the last member in Eq. (A48)

$$
\begin{aligned}
& I_{\mathrm{int}}=e \int \frac{d \omega}{2 \pi}\left(f_{\ell}-f_{r}\right) \Delta^{2} i_{\ell} i_{r} j_{\ell} j_{r} D_{0}^{R}\left[1+\alpha^{A} D_{0}^{A}\left(i_{\ell}^{2}+i_{r}^{2}\right)\right] \\
& \times \cos \Phi\left(G_{d d}^{R}+G_{d d}^{A}+\left(\Sigma_{\mathrm{ext}}^{R}+\Sigma_{\mathrm{ext}}^{A}\right) \frac{G_{d d}^{R}-G_{d d}^{A}}{\Sigma_{\mathrm{ext}}^{R}-\Sigma_{\mathrm{ext}}^{A}}\right) . \quad(\mathrm{A} 51)
\end{aligned}
$$

An important aspect of the result for the current through the interferometer, Eq. (A48), is that it is an even function of the flux $\Phi$, since both $G_{d d}^{R, A}$ and $\Sigma_{\text {ext }}^{R, A}$ are even functions of $\Phi$. Namely, the current through the interferometer obeys the Onsager relations. ${ }^{63}$ It is interesting to note that this property is not apparent from Eq. (A47); However, once we use the relation (A45), which ensures current conservation, then the flux-parity of $I$ becomes clear.

To present the current in a more transparent manner, we write the couplings $i_{\ell}, i_{r}, j_{\ell}$, and $j_{r}$, in terms of the partial widths they induce on the localized levels of the interferometer (the one on the reference arm and the one on the dot). In general, when a localized level is coupled by a matrix element $u_{k}$ to a continuum of states of energies $\epsilon_{k}$, it becomes a resonance of width

$$
\Gamma(\omega)=\pi \sum_{k}\left|u_{k}\right|^{2} \delta\left(\omega-\epsilon_{k}\right) .
$$

Making use of the matrix elements, Eqs. (5), in conjunction with Eq. (A10), we define 


$$
\gamma_{\ell}=i_{\ell}^{2} \frac{\Delta}{2 i}, \quad \gamma_{r}=i_{r}^{2} \frac{\Delta}{2 i}
$$

for the partial widths on the reference site, and

$$
\Gamma_{\ell}=j_{\ell}^{2} \frac{\Delta}{2 i}, \quad \Gamma_{r}=j_{r}^{2} \frac{\Delta}{2 i},
$$

for the partial widths on the quantum dot. In accordance with the wide-band approximation used to obtain the current Eq. (A48), we also neglect the frequencydependence of those widths. As a result, the various parameters appearing in Eqs. (A48), (A49), (A50), and (A51) can be put in the following forms. Firstly, we consider the prefactor in the expression for $I_{\text {ref }}$,

$$
\Delta^{2}\left|i_{\ell}^{2} i_{r}^{2} D_{0}^{R}\right|^{2}=-2 i \frac{\gamma_{\ell} \gamma_{r}}{\gamma_{\ell}+\gamma_{r}}\left(D_{0}^{R}-D_{0}^{A}\right) \equiv-T_{B},
$$

where $T_{B}$ is the transmission coefficient of the reference arm of the interferometer (when decoupled from the quantum dot). In the wide-band approximation (in which the energy is taken to be at the middle of the band)

$$
T_{B}=\frac{4 \gamma_{\ell} \gamma_{r}}{\epsilon_{0}^{2}+\left(\gamma_{\ell}+\gamma_{r}\right)^{2}}
$$

Secondly, the prefactor in the expression for $I_{\mathrm{dot}}$ becomes

$$
\Delta^{2} j_{\ell}^{2} j_{r}^{2}\left|1+\alpha^{R} D_{0}^{R}\left(i_{\ell}^{2}+i_{r}^{2}\right)\right|^{2} \equiv-4 \Gamma_{\ell} \Gamma_{r} X_{B},
$$

with $X_{B}$ given in Eq. (15). Finally, the coefficient in $I_{\text {int }}$ is

$$
\begin{aligned}
& \Delta^{2} i_{\ell} i_{r} j_{\ell} j_{r} D_{0}^{R}\left(1+\alpha^{A} D_{0}^{A}\left(i_{\ell}^{2}+i_{r}^{2}\right)\right) \\
= & -2 \operatorname{sgn}\left(\epsilon_{0}\right) \sqrt{T_{B} \Gamma_{\ell} \Gamma_{r} X_{B}} .
\end{aligned}
$$

For the sake of completeness, we add here the 'external' self-energy of the dot Green function, expressed in terms of the partial resonance widths,

$$
\begin{aligned}
\Sigma_{\mathrm{ext}}^{R} & =-i\left(\Gamma_{\ell}+\Gamma_{r}-\frac{T_{B}\left(\gamma_{\ell}+\gamma_{r}\right)}{4} Z_{B}\right) \\
& +\operatorname{sgn}\left(\epsilon_{0}\right) \frac{Z_{B}}{2} \sqrt{T_{B} \gamma_{\ell} \gamma_{r} X_{B}}
\end{aligned}
$$

with

$$
Z_{B}=\frac{\Gamma_{\ell}}{\gamma_{r}}+\frac{\Gamma_{r}}{\gamma_{\ell}}-2 \cos \Phi \sqrt{\frac{\Gamma_{\ell} \Gamma_{r}}{\gamma_{\ell} \gamma_{r}}} .
$$

It is thus seen that both the imaginary and the real parts of $\Sigma_{\text {ext }}$ depend on the flux threading the interferometer, through the interference term $Z_{B}$. This expression for the external self-energy differs from the one reported in Ref. 22, in which the imaginary part of $\Sigma_{\text {ext }}^{R}$ is independent of the flux, while its real part vanishes for $\Phi=\pi / 2$. Although the details of $\Sigma_{\text {ext }}^{R}$ are necessarily model-dependent, the result given there, which apparently neglects any scattering on the reference arm, is obviously rather restricted to a very specific situation.
Inserting the results (A55), (A57), and (A58) into Eqs. (A49), (A50), and (A51) yields our final result for the current through the interferometer, Eq. (14), where for simplicity we have chosen the sign of the on-site energy on the reference site to be positive. We note that this result is not the same as the ones given in Refs. 21 and 22, which neglected the scattering on the reference site. On the other hand, our expression reduces to the result obtained from a straightforward calculation (that does not employ the Keldysh technique), for an interaction-free system, as will be shown below.

When there are no interactions on the dot, then using Eqs. (A43), and denoting the interaction-free current by $I^{0}$, one has

$$
\begin{aligned}
I^{0}= & e \int \frac{d \omega}{2 \pi}\left(f_{r}-f_{\ell}\right)\left\{T_{B}\left|1+G_{d d}^{R 0} \Sigma_{\text {ext }}^{R}\right|^{2}\right. \\
+ & 4 \Gamma_{\ell} \Gamma_{r} X_{B}\left|G_{d d}^{R 0}\right|^{2}+2 \sqrt{T_{B} \Gamma_{\ell} \Gamma_{r} X_{B}} \cos \Phi \\
& \left.\times\left(G_{d d}^{R 0}\left(1+\Sigma_{\mathrm{ext}}^{A} G_{d d}^{A 0}\right)+c c\right)\right\} .
\end{aligned}
$$

Noting now that

$$
\left(G_{d d}^{R 0}\right)^{-1}+\Sigma_{\text {ext }}^{R} \equiv\left(\mathcal{G}^{0}\right)^{-1} \equiv \omega-\epsilon_{d}
$$

is a real function, $I^{0}$ can be written as

$$
\begin{aligned}
I^{0}= & e \int \frac{d \omega}{2 \pi}\left(f_{r}-f_{\ell}\right)\left|G_{d d}^{R 0}\right|^{2} \\
& \times\left|\sqrt{T_{B}}\left(\mathcal{G}^{0}\right)^{-1} e^{i \Phi}+2 \sqrt{\Gamma_{\ell} \Gamma_{r} X_{B}}\right|^{2} .
\end{aligned}
$$

This result reproduces the one found using scatteringmatrix description. ${ }^{24}$

\section{The circulating current}

In order to calculate the current circulating around the interferometer, we consider the quantity $\left(I_{1}-I_{2}-I_{3}+\right.$ $\left.I_{4}\right) / 2$ employing Eqs. (A27) and (A34), and then take its antisymmetric part with respect to the flux. Clearly the first term in Eq. (A34) will eventually disappear, since it is independent of $\Phi$. Therefore, we will not include that term. We have

$$
\begin{aligned}
& \frac{I_{1}-I_{2}-I_{3}+I_{4}}{2} \\
= & e \int \frac{d \omega}{2 \pi}\left\{2 i \frac{f_{\ell}+f_{r}}{2}\left[\frac{\partial \Sigma_{\mathrm{ext}}^{A}}{\partial \Phi} G_{d d}^{A}-c c\right]\right. \\
+ & \frac{1}{2}\left[\alpha^{R} J_{\ell}^{R}(-\Phi)\left(2 j_{\ell}-\left(J_{\ell}^{R}(-\Phi)\right)^{*}\right)-c c\right] \\
& \times\left[G_{d d}^{<}+f_{\ell}\left(G_{d d}^{R}-G_{d d}^{A}\right)\right] \\
- & \frac{1}{2}\left[\alpha^{R} J_{r}^{R}(-\Phi)\left(2 j_{r}-\left(J_{r}^{R}(-\Phi)\right)^{*}\right)-c c\right] \\
& \times\left[G_{d d}^{<}+f_{r}\left(G_{d d}^{R}-G_{d d}^{A}\right)\right] \\
+ & \left(f_{r}-f_{\ell}\right) \Delta\left[G _ { d d } ^ { R } \left(i_{\ell}^{2} i_{r}^{2} D_{0}^{R} D_{0}^{A}\left(\Delta \Sigma_{\mathrm{ext}}^{R}-\alpha^{R} \alpha^{A}\left(j_{\ell}^{2}+j_{r}^{2}\right)\right)\right.\right.
\end{aligned}
$$




$$
\begin{aligned}
& -i_{\ell} i_{r} j_{\ell} j_{r} \cos \Phi\left(\alpha^{A} D_{0}^{A}+\alpha^{R} D_{0}^{R}\right) \\
& \left.\left.\left.-\left|\alpha^{R} D_{0}^{R}\right|^{2} i_{\ell} i_{r} j_{\ell} j_{r}\left(i_{r}^{2} e^{-i \Phi}+i_{\ell}^{2} e^{i \Phi}\right)\right)-c c\right]\right\}
\end{aligned}
$$

where in order to obtain the first term here we have used Eq. (A37). Clearly, when the system is unbiased and the distribution functions $f_{\ell}$ and $f_{r}$ are the thermal ones [see Eq. (A40)], only that term survives. In order to explore the situation in which the system is biased, we proceed as follows. Firstly, we consider the contribution of the last square brackets, multiplying the difference $f_{r}-f_{\ell}$. The antisymmetric part with respect to $\Phi$ is just

$$
i \sin \Phi G_{d d}^{R} i_{\ell} i_{r} j_{\ell} j_{r}\left|\alpha^{R} D_{0}^{R}\right|^{2}\left(i_{r}^{2}-i_{\ell}^{2}\right)-c c .
$$

Next we consider the two terms involving the combinations $G_{d d}^{<}+f_{\ell}\left(G_{d d}^{R}-G_{d d}^{A}\right)$ and $G_{d d}^{<}+f_{r}\left(G_{d d}^{R}-G_{d d}^{A}\right)$, using Eqs. (A46). The sum of these two terms becomes

$$
\begin{aligned}
& \frac{\Delta}{2}\left(f_{r}-f_{\ell}\right) \frac{G_{d d}^{R}-G_{d d}^{A}}{\Sigma_{\mathrm{ext}}^{R}-\Sigma_{\mathrm{ext}}^{A}}\left[| J _ { r } ^ { R } ( \Phi ) | ^ { 2 } \left(\Delta\left|J_{\ell}^{R}(-\Phi)\right|^{2}\right.\right. \\
+ & \left.2 \alpha^{R} j_{\ell} J_{\ell}^{R}(-\Phi)-2 \alpha^{A} j_{\ell}\left(J_{\ell}^{R}(-\Phi)\right)^{*}\right) \\
+ & \left|J_{\ell}^{R}(\Phi)\right|^{2}\left(\Delta\left|J_{r}^{R}(-\Phi)\right|^{2}\right. \\
+ & \left.\left.2 \alpha^{R} j_{r} J_{r}^{R}(-\Phi)-2 \alpha^{A} j_{r}\left(J_{r}^{R}(-\Phi)\right)^{*}\right)\right] .
\end{aligned}
$$

The antisymmetric part with respect to $\Phi$ is

$$
\begin{aligned}
& \frac{\Delta}{2}\left(f_{r}-f_{\ell}\right) \frac{G_{d d}^{R}-G_{d d}^{A}}{\sum_{\mathrm{ext}}^{R}-\Sigma_{\mathrm{ext}}^{A}} i_{\ell} i_{r} j_{\ell} j_{r} 2 i \sin \Phi \\
& \times \alpha^{R} \alpha^{A}\left[D_{0}^{R} D_{0}^{A}\left(\Sigma_{\mathrm{ext}}^{R}+\Sigma_{\mathrm{ext}}^{A}\right)\left(i_{r}^{2}-i_{\ell}^{2}\right)\right. \\
+ & \left.2 D_{0}^{A}\left(1+\alpha^{R} D_{0}^{R}\left(i_{\ell}^{2}+i_{r}^{2}\right)\right)\left(j_{r}^{2}-j_{\ell}^{2}\right)\right] .
\end{aligned}
$$

Inspecting Eqs. (A65) and (A67), we see that once the interferometer is biased, there appear terms in the circulating current resulting from asymmetries in the couplings. Collecting the results above, the circulating current, $I_{\text {cir }}$, can be written as

$$
I_{\text {cir }}=I_{p c}+I_{a} .
$$

Here, $I_{p c}$ denotes the part of the circulating current which survives even when the system is un-biased, and is therefore termed 'persistent current',

$$
I_{p c}=e \int \frac{d \omega}{i \pi} \frac{f_{\ell}+f_{r}}{4}\left[\frac{\partial \Sigma_{\mathrm{ext}}^{R}}{\partial \Phi} G_{d d}^{R}-c c\right] .
$$

The additional circulating current, that arises only when the system is biased and there are asymmetries in the couplings is denoted $I_{a}$,

$$
\begin{gathered}
I_{a}=e i_{\ell} i_{r} j_{\ell} j_{r}(i \sin \Phi) \int \frac{d \omega}{4 \pi}\left(f_{r}-f_{\ell}\right) \Delta \\
\times\left[( i _ { r } ^ { 2 } - i _ { \ell } ^ { 2 } ) | \alpha ^ { R } D _ { 0 } ^ { R } | ^ { 2 } \left(G_{d d}^{R}+G_{d d}^{R}\right.\right. \\
\left.+\left(\Sigma_{\mathrm{ext}}^{R}+\Sigma_{\mathrm{ext}}^{A}\right) \frac{G_{d d}^{R}-G_{d d}^{A}}{\Sigma_{\mathrm{ext}}^{R}-\Sigma_{\mathrm{ext}}^{A}}\right)+2\left(j_{r}^{2}-j_{\ell}^{2}\right)\left|\alpha^{R}\right|^{2} \\
\left.\times D_{0}^{A}\left(1+\alpha^{R} D_{0}^{R}\left(i_{\ell}^{2}+i_{r}^{2}\right)\right) \frac{G_{d d}^{R}-G_{d d}^{A}}{\sum_{\mathrm{ext}}^{R}-\Sigma_{\mathrm{ext}}^{A}}\right] .
\end{gathered}
$$

In particular, when the system is free of interactions, we use Eqs. (A62), and the analogous expression for $D_{0}^{R}$,

$$
\omega-\epsilon_{0} \equiv\left(\mathcal{D}^{0}\right)^{-1} \equiv\left(D_{0}^{R}\right)^{-1}+\alpha^{R}\left(i_{\ell}^{2}+i_{r}^{2}\right),
$$

to find that the 'asymmetric' part of the interaction-free circulating current is given by

$$
\begin{aligned}
I_{a}^{0}= & e i_{\ell} i_{r} j_{\ell} j_{r}(2 i \sin \Phi) \int \frac{d \omega}{4 \pi}\left(f_{r}-f_{\ell}\right) \Delta\left|\alpha^{R} D_{0}^{R} G_{d d}^{R 0}\right|^{2} \\
& \times\left[\left(i_{r}^{2}-i_{\ell}^{2}\right)\left(\mathcal{G}^{0}\right)^{-1}+\left(j_{r}^{2}-j_{\ell}^{2}\right)\left(\mathcal{D}^{0}\right)^{-1}\right] . \quad(\mathrm{A} 72)
\end{aligned}
$$

In the main text we omit this part of the circulating current, that arises from the coupling asymmetries, and consider only the term $I_{p c}$. Moreover, when the potential difference across the interferometer is small (namely, the system is in the linear response regime), one may neglect this difference altogether in the sum $f_{\ell}+f_{r}$, and replace the electron distributions by the thermal distribution one, Eq. (A40). Note that then, the relation (A45) becomes exact, and therefore the result (A69) does not rely on the wide-band approximation. This is quite fortunate, since the persistent current, as opposed to the transport current, requires integration over the entire band. Hence, using for it an approximation which is valid at a narrow range around the common Fermi energy is not easily justifiable.

${ }^{1}$ Y. Imry, Introduction to Mesoscopic Physics (Oxford University Press, Oxford 1997; 2nd edition 2002).

${ }^{2}$ Y. Aharonov and D. Bohm, Phys. Rev. 115, 485 (1959).

${ }^{3}$ A. Yacoby, M. Heiblum, D. Mahalu, and H. Shtrikman, Phys. Rev. Lett. 74, 4047 (1995).

${ }^{4}$ R. Schuster, E. Buks, M. Heiblum, D. Mahalu, V. Umansky, and H. Shtrikman, Nature (London) 385, 417 (1997); E. Buks, R. Schuster, M. Heiblum, D. Mahalu, and V. Umansky, Nature (London) 391, 871 (1998).

${ }^{5}$ D. Sprinzak, E. Buks, M. Heiblum, and H. shtrikman, Phys. Rev. Lett. 84, 5820 (2000).

${ }^{6}$ W. G. van der Wiel, S. De Franceschi, T. Fujisawa, J. M. Elzerman, S. Tarucha, and L. P. Kouwenhoven, Science 289, 2105 (2000).

${ }^{7}$ Y. Ji, M. Heiblum, D. Sprinzak, D. Mahalu, and H. Shtrikman, Science 290, 779 (2000); Y. Ji, M. Heiblum, and H. Shtrikman, Phys. Rev. Lett. 88, 076601 (2002).

${ }^{8}$ A. W. Holleitner, C. R. Decker, H. Qin, K. Eberl, and R. H. Blick, Phys. Rev. Lett. 87, 256802 (2001). 
${ }^{9}$ A. Fuhrer, S. Lüscher, T. Ihn, T. Heinzel, K. Ensslin, W. Wegscheider, and M. Bichler, Nature (London) 413, 822 (2001); U. F. Keyser, C. Fühner, S. Borck, R. J. Haug, M. Bichler, G. Abstreiter, and W. Wegscheider, Phys. Rev. Lett. 90, 196601 (2003); M. Sigrit, A. Fuhrer, T. Ihn, K. Ensslin, S. E. Ulloa, W. Wegscheider, and M. Bichler, condmat/0308223 (2003).

${ }^{10}$ K. Kobayashi, H. Aikawa, S. Katsumoto, and Y. Iye, Phys. Rev. Lett. 88, 256806 (2002); K. Kobayashi, H. Aikawa, S. Katsumoto, and Y. Iye, J. Phys. Soc. Jpn. 71, L2094 (2002).

${ }^{11}$ Y. Gefen, Y. Imry, and M. Ya. Azbel, Phys. Rev. Lett. 52, 129 (1984).

${ }^{12}$ M. Büttiker, Y. Imry, R. Landauer, and S. Pinhas, Phys. Rev. B 31, 6207 (1985).

13 O. Entin-Wohlman, C. Hartzstein, and Y. Imry, Phys. Rev. B 34, 921 (1986).

${ }^{14}$ R. A. Webb, S. Washburn, C. P. Umbach, and R. B. Laibowitz, Phys. Rev. Lett. 54, 2696 (1985).

${ }^{15}$ G. Hackenbroich and H. A. Weidenmüller, Phys. Rev. Lett. 76, 110 (1996); G. Hackenbroich, Phys. Rep. 343, 463 (2001).

${ }^{16}$ C. Bruder, R. Fazio, and H. Schoeller, Phys. Rev. Lett. 76, 114 (1996).

${ }^{17}$ H.-W. Lee, Phys. Rev. Lett. 82, 2358 (1999).

18 T. Taniguchi and M. Büttiker, Phys. Rev. B 60, 13814 (1999).

19 A. L. Yeyati and M. Büttiker, Phys. Rev. B 62, 7307 (2000).

${ }^{20}$ U. Gerland, J. von Delft, T. A. Costi, and Y. Oreg, Phys. Rev. Lett. 84, 3710 (2000).

${ }^{21}$ B. R. Bulka and P. Stefański, Phys. Rev. Lett. 86, 5128 (2001).

${ }^{22}$ W. Hofstetter, J. König, and H. Schoeller, Phys. Rev. Lett. 87, 156803 (2001).

23 J. König and Y. Gefen, Phys. Rev. Lett. 86, 3855 (2001); Phys. Rev. B 65, 045326 (2002).

${ }^{24}$ O. Entin-Wohlman, A. Aharony, Y. Imry, Y. Levinson and A. Schiller, Phys. Rev. Lett. 88, 166801 (2002); O. EntinWohlman, A. Aharony, B. I. Halperin, and Y. Imry, Phys. Rev. B 66, 115311 (2002); A. Aharony, O. Entin-Wohlman, and Y. Imry, Phys. Rev. Lett. 90, 156802 (2003).

${ }^{25}$ H. A. Weidenmüller, Phys. Rev. B 65, 245322 (2002).

${ }^{26}$ L. Pauling, J. Chem. Phys. 4, 673 (1936).

${ }^{27}$ F. London, J. Phys. Radium 8, 397 (1937).

${ }^{28}$ I. O. Kulik, JETP Lett. 11, 275 (1970).

${ }^{29}$ M. Büttiker, Y. Imry, and R. Landauer, Phys. Lett. A 96, 3365 (1985).

${ }^{30}$ L. P. Lévy, G. Dolan, J. Dunsmuir, and H. Bouchiat, Phys. Rev. Lett. 64, 2074 (1990).

${ }^{31}$ V. Chandrasekhar, R. A. Webb, M. J. Brady, M. B. Ketchen, W. J. Gallagher, and A. Kleinsasser, Phys. Rev. Lett. 67, 3578 (1991).

32 D. Mailly, C. Chapelier, and A. Benoit, Phys. Rev. Lett. 70, 2020 (1993).

${ }^{33}$ E. M. Q. Jariwala, P. Mohanty, M. B. Ketchen, and R. A. Webb, Phys. Rev. Lett. 86, 1594 (2001).

${ }^{34}$ W. Rabaud, L. Saminadayar, D. Mailly, K. Hasselbach, A. Benoit, and B. Etienne, Phys. Rev. Lett. 86, 3124 (2001).

${ }^{35}$ R. Deblock, R. Bel, B. Reulet, H. Bouchiat, and D. Mailly,
Phys. Rev. Lett. 89, 206803 (2002).

${ }^{36}$ A. Lorke, R. Luyken, A. O. Govorov, J. P. Kotthaus, J. M. Garcia, and P. M. Petroff, Phys. Rev. Lett. 84, 2223 (2000).

37 R. Warburton, C. Schäflein, D. Haft, F. Bickel, A. Lorke, K. Kaprai, J. M. Garcia, W. Schoenfeld, and P. M. Petroff, Nature (London) 405, 926 (2000).

${ }^{38}$ M. Bayer, M. Korkusinski, P. Hawrylak, T. Gutbrod, M. Michel, and A. Forchel, Phys. Rev. Lett. 90, 186801 (2003).

${ }^{39}$ M. Büttiker and C. A. Stafford, Phys. Rev. Lett. 76, 495 (1996).

${ }^{40}$ S. Y. Cho, K. Kang, C. K. Kim, and C-M. Ryu, Phys. Rev. B 64, 033314 (2001).

${ }^{41}$ G-H. Ding and B. Dong, Phys. Rev. B 67, 195327 (2003).

${ }^{42}$ V. E. Kravtsov and V. I. Yudson, Phys. Rev. Lett. 70, 210 (1993).

43 Y. Galperin and O. Entin-Wohlman, Phys. Rev. B 54, 9346 (1996).

${ }^{44}$ M. Moskalets and M. Büttiker, Phys. Rev. B 66, 245321 (2002).

45 O. L. Chalaev and V. E. Kravtsov, Phys. Rev. Lett. 89, 176601 (2002).

${ }^{46}$ V. Ferrari, G. Chiappe, E. V. Anda, and M. D. Davidovich, Phys. Rev. Lett. 82, 5088 (1999).

${ }^{47}$ K. Kang and S-C. Shin, Phys. Rev. Lett. 85, 5619 (2000); K. Kang, S. Y. Cho, J-J. Kim, and S-C. Shin, Phys. Rev. B 63, 113304 (2001).

${ }^{48}$ I. Affleck and P. Simon, Phys. Rev. Lett. 86, 2854 (2001).

${ }^{49}$ H. Hu, G-M. Zhang, and L. Yu, Phys. Rev. Lett. 86, 5558 (2001).

${ }^{50}$ H.-P. Eckle, H. Johannesson, and C. A. Stafford, Phys. Rev. Lett. 87, 016602 (2001).

${ }^{51}$ P. Mohanty, Ann. Phys. (Leipzig) 8, 549 (1999).

${ }^{52}$ P. Schwab, Eur. Phys. J. B18, 189 (2000).

${ }^{53}$ V. E. Kravtsov and B. L. Altshuler, Phys. Rev. Lett. 84, 3394 (2000).

${ }^{54}$ P. Cedraschi, V. V. Ponomarenko and M. Büttiker, Phys. Rev. Lett. 84, 346 (2000); P. Cedraschi and M. Büttiker, Phys. Rev. B 63, 165312 (2001).

${ }^{55}$ T. Holstein, Ann. Phys. (N. Y.) 8, 343 (1959).

${ }^{56}$ O. Entin-Wohlman, Y. Imry, A. G. Aronov, and Y. Levinson, Phys. Rev. B 51, 11584 (1995).

${ }^{57}$ B. A. Glavin, V. A. Kochelap, and T. L. Linnik, JETP Lett. 51, 191 (2000); S. A. Cavill, L. J. Challis, A. J. Kent, F. F. Ouali, A. V. Akimov, and M. Henini, Phys. Rev. B 66, 235320 (2002).

${ }^{58}$ R. G. Mani, J. H. Smet, K. von Klitzing, V. Narayanamurti, W. B. Johnson, and V. Umansky, Nature (London) 420, 646 (2002); cond-mat/0305507.

${ }^{59}$ L. I. Glazman and R. I. Shekhter, Sov. Phys. JETP 67, 163 (1988).

${ }^{60}$ N. S. Wingreen, K. W. Jacobsen, and J. W. Wilkins, Phys. Rev. Lett. 61, 1396 (1988); Phys. Rev. B 40, 11834 (1989).

${ }^{61}$ A-P. Jauho, N. S. Wingreen, and Y. Meir, Phys. Rev. B 50, 5528 (1994).

${ }^{62}$ L. V. Keldysh, Sov. Phys. JETP 20, 1018 (1965); D. C. Langreth, in Linear and Nonlinear Electron Transport in Solids, Vol. 17 of Nato Advanced Study Institute, Series B: Physics, edited by J. T. Devreese and V. E. Van Doren (Plenum, New York, 1976). 
63 The role of the Onsager relations in flux-sensitive transport is discussed by M. Büttiker and Y. Imry, J. Phys. C18, L467 (1985) and by M. Büttiker, Phys. Rev. Lett. 57, 1761 (1986).

${ }^{64}$ O. Entin-Wohlman, A. Aharony, Y. Imry, and Y. Levinson, J. Low Temp. Phys. 126, 1251 (2002).

${ }^{65}$ M. Büttiker, Phys. Rev. B 32, 1846 (1985).

${ }^{66}$ E. Akkermans, A. Auerbach, J. E. Avron, and B. Shapiro, Phys. Rev. Lett. 66, 76 (1991).

${ }^{67}$ P. A. Mello, Phys. Rev. B 47, 16358 (1993).

${ }^{68}$ O. Entin-Wohlman, Y. Imry, and A. Aharony, Phys. Rev. Lett. 91, 046802 (2003).

${ }^{69}$ K. Haule and J. Bonča, Phys. Rev. B 59, 13087 (1999).
${ }^{70}$ Y. Imry, cond-mat/0202044 (2002).

${ }^{71}$ M. Jonson, Phys. Rev. B 39, 5924 (1989).

${ }^{72}$ L. E. F. Foa Torres, H. M. Patawski, and S. S. Makler, Phys. Rev. B 64, 193304 (2001).

73 T. Holstein, Phys. Rev. 124, 1329 (1961).

${ }^{74}$ A. Miller and E. Abrahams, Phys. Rev. 120, 745 (1960).

${ }^{75}$ A. Stern, Y. Aharonov, and Y. Imry, Phys. Rev. A 41, 3436 (1990).

${ }^{76}$ A. A. Burkov and L. Balents, Phys. Rev. Lett. 91, 057202 (2003).

77 O. Entin-Wohlman, A. G. Aronov, Y. Levinson, and Y. Imry, Phys. Rev. Lett. 75, 4094 (1995). 